\title{
Temperature Fluctuations in Dusty Fluid Homogeneous Turbulence at Four Point Correlations
}

\author{
Abdul Malek, Md. Abul Kalam Azad, Md. Abu Bkar Pk \\ Department of Applied Mathematics, University of Rajshahi, Rajshahi, Bangladesh \\ Email address: \\ am.math.1970@gmail.com (A. Malek), azad267@gmail.com (Md. A. K. Azad), abubakarpk_ru@yahoo.com (Md. A. Bkar Pk) \\ To cite this article: \\ Abdul Malek, Md. Abul Kalam Azad, Md. Abu Bkar Pk. Temperature Fluctuations in Dusty Fluid Homogeneous Turbulence at Four Point \\ Correlations. American Journal of Applied Mathematics. Vol. 5, No. 5, 2017, pp. 132-144. doi: 10.11648/j.ajam.20170505.12
}

Received: May 8, 2017; Accepted: May 25, 2017; Published: September 30, 2017

\begin{abstract}
In this study the temperature fluctuations in dusty fluid homogeneous turbulence prior to the final period is considered by using the four point correlation equations for fluctuating quantities in the flow field. The correlation equations are converted into spectral form by their Fourier-transform. The set of equations are made to determinate by neglecting the quintuple correlations in comparison to the fourth- order correlation terms. Finally by integration of the energy spectrum over all wave numbers and have obtained the energy equation of decaying of temperature fluctuations in dusty fluid homogeneous turbulence for four point correlations. The obtained results have been shown by graphically at different Prandtl no. and at the different state of temperature. It is also determined the values of the constant appear at the energy equation by using the values of the parameters which exist in it for different fluids. It has also been shown the effects of the parameters by graphically.
\end{abstract}

Keywords: Four-Point Correlation, Decay, Temperature Fluctuations, Final Period, Dust Particles, Prandtl No

\section{Introduction}

Very recently the motion in dusty fluid in homogeneous turbulence problem is developed rapidly. The motion of dusty fluid is observed in the movement of dust-laden air, in a gas cooling system, in the tidal rivers and in other fluids. The behavior of dust particles in a turbulent flow depend on the concentration of the particles, the size of the particles and quantities of the particles with respect to scale of the turbulent fluid.

In the past, some researchers had done their research considering dust particles in turbulence and $\mathrm{MHD}^{1}$ turbulent flow. Corrsin [1] considered on the spectrum of isotropic temperature fluctuations in isotropic turbulence. Deissler [2, $3]$ developed a theory on decay of homogeneous turbulence for times before the final period for three and four point correlation. In the next, Loeffler and Deissler [4] extended their theory for the case of decaying of temperature fluctuations in homogeneous turbulence. Saffman [5] derived an equation that describes the fluid containing small dust particles which is applicable laminar flow as well as turbulent flow. Kishore and Sinha [6] studied the statistical

$1 \mathrm{MHD}=$ Magneto-hydro Dynamic. theory of decay process of homogeneous hydromagnetic turbulance, Kishorand Golsefied [7] have done their research on theEffect of coriolis force on acceleration covariance in MHD turbulent flow of a dusty incompressible fluid. Kishor and Sarker [8] also studied the rate of change of vorticity covariance in MHD turbulent flow of dusty incompressible fluid. Recently, Azad and Sarker [9, 10, 12] calculated a results on decaying of MHD turbulence before the final period for the case of multi-point and multi-time in presence of dust particle taking Coriolis force. Azad et al $[11,13]$ studied the decay of temperature fluctuations in dusty fluid MHD turbulence in a rotating system before the final period. Sarker et al [14] have been done their work on first order reactant in MHD turbulence before the final Period of decay for the case of multi-point and multi-time in presence of dust particles. Azad et al $[15,16]$ had done their research on the decay law of temperature fluctuations in dusty fluid MHD turbulence before the final period. They also had done their work on joint distribution function of velocity, temperature and concentration in convective dusty fluid tubulent flow. Azad and Mumtahinah [17, 18] considered the decay of temperature fluctuations in dusty fluid homogeneous turbulence prior to final period with Coriolis force. Bkar et al [19] studied on 4-point correlations of dusty fluid MHD 
turbulent flow in a $1^{\text {st }}$ order chemical reaction. Azad et al [20, $21,22,23]$ had done their work on the effect of chemical reaction on statistical theory of dusty fluid MHD turbulent flow for certain variables at three-point correlation. Alam et al [24] Study the Effect of Chemical Reaction and Magnetic Field on Free Convection Boundeary Layer Flow of Heat and MassTransfer with Variable Prandtl Number. Most recently Mamun et al [25] also studiedthe effect of 1st order chemical reaction in convective dusty fluids turbulent flow for threepoint Joint Distribution Functions.

The above researchers have considered two and three point correlation equations and solved these equations after neglecting the fourth and higher order correlation terms. But in this work we have solved for four point correlations equations after neglecting the quintuple and higher order correlation terms for dusty fluid turbulent flow.

The main purpose of the present study is to find a possible solution for the dynamics of decaying the temperature fluctuation in homogeneous dusty fluid turbulence for four point correlation. Through this study, we have obtained energy equation of decaying of temperature fluctuations in dusty fluid homogeneous turbulence at times before the final period for four- point correlation systems which comes out in the following form

$$
\left\langle T^{2}\right\rangle=W\left(t-t_{0}\right)^{-3 / 2}+X\left(t-t_{0}\right)^{-5}+Y\left(t-t_{1}\right)^{-\frac{7}{2}} \exp \left[l\left(t-t_{1}\right)\right]+Z\left(t-t_{1}\right)^{-7} \exp \left[l\left(t-t_{1}\right)\right],
$$

Where $\left\langle T^{2}\right\rangle$ denotes the total energy and $\mathrm{t}$ is the time, $\mathrm{W}, \mathrm{X}$,

$\mathrm{Y}$ and $\mathrm{Z}$ are arbitrary constants determined by initial conditions. Through this study we have tried to show the effect of varying Prandtl no. by graphically. In this study, it observed that the energy more decays than the clean fluid.

\section{Four Point Correlation and Spectral Equations}

In order to find the four point correlations and spectral equations for single time and take the momentum equation of turbulence at the point $\mathrm{P}$ and the energy equation of Temperature fluctuation for four point correlations in presence of dust particle at $P^{\prime}, P^{\prime \prime}$ and $P^{\prime \prime \prime}$ with position vectors $\hat{r}, \hat{r}^{\prime}$ and $\hat{r}^{\prime \prime}$

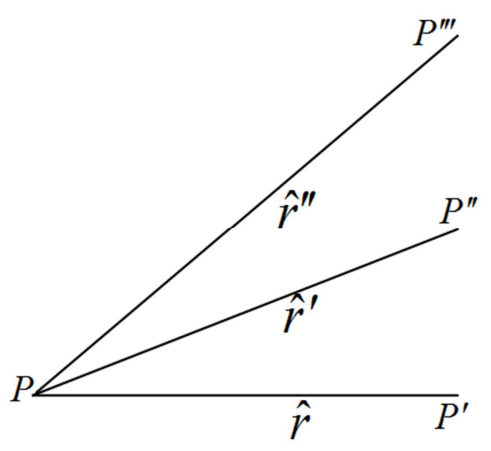

Vector configuration for four point correlation equations.

$$
\begin{gathered}
\frac{\partial u_{j}}{\partial t}+\frac{\partial\left(u_{j} u_{i}\right)}{\partial x_{i}}=-\frac{1}{\rho}+\frac{v}{P_{r}} \frac{\partial^{2} u_{j}}{\partial x_{i} \partial x_{i}}+f\left(u_{i}-v_{i}\right) \\
\frac{\partial T^{\prime}}{\partial t}+u_{i}^{\prime} \frac{\partial T^{\prime}}{\partial x_{i}}=\frac{v}{p_{r}} \frac{\partial^{2} T^{\prime}}{\partial x_{i}^{\prime} \partial x_{i}^{\prime}} \\
\frac{\partial T^{\prime \prime}}{\partial t}+u_{i}^{\prime \prime} \frac{\partial T^{\prime \prime}}{\partial x_{i}}=\frac{v}{p_{r}} \frac{\partial^{2} T^{\prime \prime}}{\partial x_{i}^{\prime \prime} x_{i}^{\prime \prime}} \\
\frac{\partial T^{\prime \prime \prime}}{\partial t}+u_{i}^{\prime \prime \prime} \frac{\partial T^{\prime \prime \prime}}{\partial x_{i}^{\prime \prime \prime}}=\frac{v}{p_{r}} \frac{\partial^{2} T^{\prime \prime \prime}}{\partial x_{i}^{\prime \prime} \partial x_{i}^{\prime \prime \prime}}
\end{gathered}
$$

Where, $T, T^{\prime}, T^{\prime \prime}, T^{\prime \prime \prime}$ denote temperature at points $P, P^{\prime}, P^{\prime \prime}$ and $P^{\prime \prime \prime}, u_{i}=$ Instantaneous velocity,

$\rho=$ Fluid density, $c_{p}=$ Heat capacity at constant pressure, $k=$ Thermal conductivity,

$x_{i}=$ Space co-ordinate, $t=$ Time, $f=\frac{K N}{\rho}$ the dimensions of frequency, $\mathrm{K}=$ Stock's drug resistance, $\mathrm{N}=$ Constant number density of dust particles, $v_{i}=$ velocity component of dust particles and the repeated subscripts are assumed from 1 to 3 .

Multiplying equation (1) by $T_{i}^{\prime} T_{j}^{\prime \prime} T_{m}^{\prime \prime \prime}$ (2) by $u_{i} T_{j}^{\prime \prime} T_{m}^{\prime \prime \prime}$ (3) by $u_{i} T_{i}^{\prime} T_{m}^{\prime \prime \prime}$ (4) by $u_{i} T_{i}^{\prime} T_{j}^{\prime \prime}$ and adding these equations, after taking time averages, result is

$$
\begin{aligned}
& \frac{\partial \overline{\left(u_{j} T_{i}^{\prime} T_{j}^{\prime \prime} T_{k}^{\prime \prime \prime}\right)}}{\partial t}+\frac{\partial \overline{\left(u_{j} u_{i} T_{i}^{\prime} T_{j}^{\prime \prime} T_{k}^{\prime \prime \prime}\right)}}{\partial x_{i}}+\frac{\partial \overline{\left(u_{i} u_{i}^{\prime} T_{i}^{\prime} T_{j}^{\prime \prime} T_{k}^{\prime \prime \prime}\right)}}{\partial x_{i}^{\prime}}+\frac{\partial \overline{\left(u_{i} u_{i}^{\prime \prime} T_{i}^{\prime} T_{j}^{\prime} T_{k}^{\prime \prime \prime}\right)}}{\partial x_{i}^{\prime \prime}}+\frac{\partial\left(\overline{\left.u_{i} u_{j}^{\prime \prime \prime} T_{i}^{\prime} T_{j}^{\prime \prime} T_{k}^{\prime \prime \prime}\right)}\right.}{\partial x_{i}^{\prime \prime \prime}} \\
& =-\frac{1}{\rho} \frac{\partial \overline{\left(P T_{i}^{\prime} T_{j}^{\prime \prime} T_{k}^{\prime \prime \prime}\right)}}{\partial x_{j}}+\frac{v}{P_{r}} \frac{\partial^{2}\left(\overline{\left.u_{j} T_{i}^{\prime} T_{j}^{\prime \prime} T_{k}^{\prime \prime \prime}\right)}\right.}{\partial x_{i} \partial x_{i}}+\frac{v}{p_{r}} \frac{\partial^{2}\left(\overline{u_{i} T_{i}^{\prime} T_{j}^{\prime \prime} T_{k}^{\prime \prime \prime}}\right)}{\partial x_{i}^{\prime} \partial x_{i}^{\prime}}+\frac{v}{p_{r}} \frac{\partial^{2}\left(\overline{\left.u_{i} T_{i}^{\prime} T_{j}^{\prime \prime} T_{k}^{\prime \prime \prime}\right)}\right.}{\partial x_{i}^{\prime \prime} \partial x_{i}^{\prime \prime}}+ \\
& \frac{v}{p_{r}} \frac{\partial^{2} u_{i} T_{i}^{\prime} T^{\prime \prime} T_{k}^{\prime \prime \prime}}{\partial x_{i}^{\prime \prime \prime} \partial x_{i}^{\prime \prime \prime}}+f\left(u_{i}-v_{i}\right) T_{i}^{\prime} T_{j}^{\prime \prime} T_{m}^{\prime \prime \prime}
\end{aligned}
$$

Now we will use the transformations

$$
\frac{\partial}{\partial x_{i}^{\prime \prime \prime}}=\frac{\partial}{\partial r_{i}^{\prime \prime}}, \frac{\partial}{\partial x_{i}^{\prime \prime}}=\frac{\partial}{\partial r_{i}^{\prime}}, \frac{\partial}{\partial x_{i}^{\prime}}=\frac{\partial}{\partial r_{i}}, \frac{\partial}{\partial x_{i}}=-\left(\frac{\partial}{\partial r_{i}}+\frac{\partial}{\partial r_{i}^{\prime}}+\frac{\partial}{\partial r_{i}^{\prime \prime}}\right) \text { into equations (5) then, }
$$




$$
\begin{aligned}
& \frac{\partial \overline{\left(u_{j} T_{i}^{\prime} T_{j}^{\prime \prime} T_{k}^{\prime \prime \prime}\right)}}{\partial t}-\frac{\partial \overline{\left(u_{j} u_{i} T_{i}^{\prime} T_{j}^{\prime \prime} T_{k}^{\prime \prime \prime}\right)}}{\partial r_{i}}-\frac{\partial \overline{\left(u_{j} u_{i} T_{i}^{\prime} T_{j}^{\prime \prime} T_{k}^{\prime \prime \prime}\right)}}{\partial r_{i}^{\prime}}-\frac{\partial \overline{\left(u_{j} u_{i} T_{i}^{\prime} T_{j}^{\prime \prime} T_{k}^{\prime \prime \prime}\right)}}{\partial r_{i}^{\prime \prime}}+\frac{\partial \overline{\left(u_{i} u_{i}^{\prime} T_{i}^{\prime} T_{j}^{\prime \prime} T_{k}^{\prime \prime \prime}\right)}}{\partial r_{i}} \\
& +\frac{\partial \overline{\left(u_{i} u_{i}^{\prime \prime} T_{i}^{\prime} T_{j}^{\prime} T_{k}^{\prime \prime \prime}\right)}}{\partial r_{i}^{\prime}}+\frac{\partial\left(\overline{\left.u_{i} u_{j}^{\prime \prime \prime} T_{i}^{\prime} T_{j}^{\prime \prime} T_{k}^{\prime \prime \prime}\right)}\right.}{\partial r_{i}^{\prime \prime}}=\frac{1}{\rho}\left[\frac{\partial \overline{\left(P T_{i}^{\prime} T_{j}^{\prime \prime} T_{k}^{\prime \prime \prime}\right)}}{\partial r_{j}}+\frac{\partial \overline{\left(P T_{i}^{\prime} T_{j}^{\prime \prime} T_{k}^{\prime \prime \prime}\right)}}{\partial r_{j}^{\prime}}+\frac{\partial \overline{\left(P T_{i}^{\prime} T_{j}^{\prime \prime} T_{k}^{\prime \prime \prime}\right)}}{\partial r_{j}^{\prime \prime}}\right] \\
& +\frac{v}{P_{r}}\left[\left(1+P_{r}\right) \frac{\partial^{2}\left(\overline{u_{i} T_{i}^{\prime} T_{j}^{\prime \prime} T_{k}^{\prime \prime \prime}}\right)}{\partial r_{i} \partial r_{i}}+\left(1+P_{r}\right) \frac{\partial^{2} \overline{\left(u_{i} T_{i}^{\prime} T_{j}^{\prime} T_{k}^{\prime \prime \prime}\right)}}{\partial r_{i} \partial r_{i}^{\prime}}+\left(1+P_{r}\right) \frac{\partial^{2} \overline{\left(u_{i} T_{i}^{\prime} T^{\prime \prime} T_{k}^{\prime \prime \prime}\right)}}{\partial r_{i}^{\prime} \partial r_{i}^{\prime \prime}}\right. \\
& +2 P_{r} \frac{\partial^{2}\left(\overline{\left.u_{i} T_{i}^{\prime} T_{j}^{\prime} T_{k}^{\prime \prime \prime}\right)}\right.}{\partial r_{i} \partial r_{i}^{\prime}}+2 P_{r} \frac{\partial^{2}\left(u_{i} T_{i}^{\prime} T_{j}^{\prime} T_{k}^{\prime \prime \prime}\right)}{\partial r_{i}^{\prime} \partial r_{i}^{\prime \prime}}+2 P_{r} \frac{\partial^{2}\left(\overline{\left.u_{i} T_{i}^{\prime} T_{j}^{\prime} T_{k}^{\prime \prime \prime}\right)}\right.}{\partial r_{i} \partial r_{i}^{\prime \prime}}+f\left(u_{i}-v_{i}\right) T_{i}^{\prime} T_{j}^{\prime \prime} T_{m}^{\prime \prime \prime}
\end{aligned}
$$

Converting the equation (6) into spectral form, now we can define the following nine dimensional Fourier transforms

$$
\begin{aligned}
& \left\langle u_{i} T_{i}^{\prime}(\hat{r}) T_{j}^{\prime \prime}\left(\hat{r}^{\prime}\right) T_{m}^{\prime \prime \prime}\left(\hat{r}^{\prime \prime}\right)\right\rangle=\int_{-\infty}^{\infty} \int_{-\infty}^{\infty} \int_{-\infty}^{\infty}\left\langle\phi_{i} \gamma_{i}^{\prime}(\hat{k}) \gamma_{j}^{\prime \prime}\left(\hat{k}^{\prime}\right) \gamma_{m}^{\prime \prime \prime}\left(\hat{k}^{\prime \prime}\right)\right\rangle \exp \left[i\left(\hat{k} \cdot \hat{r}+\hat{k}^{\prime} \cdot \hat{r}^{\prime}+\hat{k}^{\prime \prime} \cdot \hat{r}^{\prime \prime}\right) d \hat{k} d \hat{k}^{\prime} d \hat{k}^{\prime \prime}\right. \\
& \left\langle u_{j} u_{i} T_{i}^{\prime}(\hat{r}) T_{j}^{\prime \prime}\left(\hat{r}^{\prime}\right) T_{m}^{\prime \prime \prime}\left(\hat{r}^{\prime \prime}\right)\right\rangle=\int_{-\infty}^{\infty} \int_{-\infty}^{\infty} \int_{-\infty}^{\infty}\left\langle\phi_{j} \phi_{i}(\hat{k}) \gamma_{i}^{\prime}(\hat{k}) \gamma_{j}^{\prime \prime}\left(\hat{k}^{\prime}\right) \gamma_{m}^{\prime \prime \prime}\left(\hat{k}^{\prime \prime}\right)\right\rangle \exp \left[i\left(\hat{k} . \hat{r}+\hat{k}^{\prime} . \hat{r}^{\prime}+\hat{k}^{\prime \prime} . \hat{r}^{\prime \prime}\right) d \hat{k}^{\prime} d \hat{k}^{\prime} d \hat{k}^{\prime \prime}\right. \\
& \left\langle u_{i} u_{i} T_{i}^{\prime}(\hat{r}) T_{j}^{\prime \prime}\left(\hat{r}^{\prime}\right) T_{m}^{\prime \prime \prime}\left(\hat{r}^{\prime \prime}\right)\right\rangle=\int_{-\infty}^{\infty} \int_{-\infty}^{\infty} \int_{-\infty}^{\infty}\left\langle\phi_{i} \phi_{i}(\hat{k}) \gamma_{i}^{\prime}(\hat{k}) \gamma_{j}^{\prime \prime}\left(\hat{k}^{\prime}\right) \gamma_{m}^{\prime \prime \prime}\left(\hat{k}^{\prime \prime}\right)\right\rangle \exp \left[i\left(\hat{k} \cdot \hat{r}+\hat{k}^{\prime} \cdot \hat{r}^{\prime}+\hat{k}^{\prime \prime} \cdot \hat{r}^{\prime \prime}\right) d \hat{k} d \hat{k}^{\prime} d \hat{k}^{\prime \prime}\right. \\
& \left\langle u_{j} u_{i}^{\prime} T_{i}^{\prime}(\hat{r}) T_{j}^{\prime \prime}\left(\hat{r}^{\prime}\right) T_{m}^{\prime \prime \prime}\left(\hat{r}^{\prime \prime}\right)\right\rangle=\int_{-\infty}^{\infty} \int_{-\infty}^{\infty} \int_{-\infty}^{\infty}\left\langle\phi_{j} \phi_{i}^{\prime}(\hat{k}) \gamma_{i}^{\prime}(\hat{k}) \gamma_{j}^{\prime \prime}\left(\hat{k}^{\prime}\right) \gamma_{m}^{\prime \prime \prime}\left(\hat{k}^{\prime \prime}\right)\right\rangle \exp \left[i\left(\hat{k} \cdot \hat{r}+\hat{k}^{\prime} \cdot \hat{r}^{\prime}+\hat{k}^{\prime \prime} \cdot \hat{r}^{\prime \prime}\right) d \hat{k}^{\prime} d \hat{k}^{\prime} d \hat{k}^{\prime \prime}\right. \\
& \left\langle u_{j} u_{i}^{\prime \prime} T_{i}^{\prime}(\hat{r}) T_{j}^{\prime \prime}\left(\hat{r}^{\prime}\right) T_{m}^{\prime \prime \prime}\left(\hat{r}^{\prime \prime}\right)\right\rangle=\int_{-\infty}^{\infty} \int_{-\infty}^{\infty} \int_{-\infty}^{\infty}\left\langle\phi_{j} \phi_{i}^{\prime \prime}(\hat{k}) \gamma_{i}^{\prime}(\hat{k}) \gamma_{j}^{\prime \prime}\left(\hat{k}^{\prime}\right) \gamma_{m}^{\prime \prime \prime}\left(\hat{k}^{\prime \prime}\right)\right\rangle \exp \left[i\left(\hat{k} \cdot \hat{r}+\hat{k}^{\prime} \cdot \hat{r}^{\prime}+\hat{k}^{\prime \prime} \cdot \hat{r}^{\prime \prime}\right) d \hat{k} d \hat{k}^{\prime} d \hat{k}^{\prime \prime}\right. \\
& \left\langle u_{j} u_{i}^{\prime \prime \prime} T_{i}^{\prime}(\hat{r}) T_{j}^{\prime \prime}\left(\hat{r}^{\prime}\right) T_{m}^{\prime \prime \prime}\left(\hat{r}^{\prime \prime}\right)\right\rangle=\int_{-\infty}^{\infty} \int_{-\infty}^{\infty} \int_{-\infty}^{\infty}\left\langle\phi_{j} \phi_{i}^{\prime \prime \prime}\left(\hat{k}^{\prime}\right) \gamma_{i}^{\prime}(\hat{k}) \gamma_{j}^{\prime \prime}\left(\hat{k}^{\prime}\right) \gamma_{m}^{\prime \prime \prime}\left(\hat{k}^{\prime \prime}\right)\right\rangle \exp \left[i\left(\hat{k} \cdot \hat{r}+\hat{k}^{\prime} \cdot \hat{r}^{\prime}+\hat{k}^{\prime \prime} . \hat{r}^{\prime \prime}\right) d \hat{k} d \hat{k}^{\prime} d \hat{k}^{\prime \prime}\right. \\
& \left\langle P T_{i}^{\prime}(\hat{r}) T_{j}^{\prime \prime}\left(\hat{r}^{\prime}\right) T_{m}^{\prime \prime \prime}\left(\hat{r}^{\prime \prime}\right)\right\rangle=\int_{-\infty}^{\infty} \int_{\infty}^{\infty} \int\left\langle\delta \gamma_{i}^{\prime}(\hat{k}) \gamma_{j}^{\prime \prime}\left(\hat{k}^{\prime}\right) \gamma_{m}^{\prime \prime \prime}\left(\hat{k}^{\prime \prime}\right)\right\rangle \exp \left[i\left(\hat{k} \cdot \hat{r}+\hat{k}^{\prime} \cdot \hat{r}^{\prime}+\hat{k}^{\prime \prime} . \hat{r}^{\prime \prime}\right) d \hat{k} d \hat{k}^{\prime} d \hat{k}^{\prime \prime}\right.
\end{aligned}
$$

Interchange of points $p^{\prime}$ and $p^{\prime \prime}, p^{\prime}$ and $p^{\prime \prime \prime}$ the subscripts $\mathrm{i}$ and $\mathrm{j} ; \mathrm{i}$ and $\mathrm{m}$ results in the relations $\overline{\left(u_{j} u_{i}^{\prime \prime} T_{i}^{\prime} T_{j}^{\prime \prime} T_{m}^{\prime \prime \prime}\right)}=\overline{\left(u_{j} u_{i}^{\prime} T_{i}^{\prime} T_{j}^{\prime \prime} T_{m}^{\prime \prime \prime}\right)}, \overline{\left(u_{j} u_{i}^{\prime \prime} T_{i}^{\prime} T_{j}^{\prime \prime} T_{m}^{\prime \prime \prime}\right)}=\overline{\left(u_{j} u_{i}^{\prime} T_{i}^{\prime} T_{j}^{\prime \prime} T_{m}^{\prime \prime \prime}\right)}$

By using equations (7) to (13), one can write equation (6) in the form

$$
\begin{aligned}
& \frac{\partial}{\partial t}\left(\overline{\phi_{l} \gamma^{\prime} \gamma^{\prime \prime} \gamma^{\prime \prime}}\right)+\frac{v}{P_{r}}\left[\left(1+P_{r}\right) K^{2}+\left(1+P_{r}\right) K^{2}+\left(1+P_{r}\right) K^{\prime \prime 2}+2 P_{r} K K^{\prime}+2 P_{r} K K^{\prime \prime}+2 P_{r} K K^{\prime \prime \prime}\right]\left(\overline{\phi_{l} \gamma^{\prime} \gamma^{\prime \prime} \gamma^{\prime \prime \prime}}\right)= \\
& i\left(K_{k}+K_{k}^{\prime}+K^{\prime \prime}\right)\left(\overline{\phi_{l} \phi_{k} \gamma_{i}^{\prime} \gamma_{j}^{\prime \prime} \gamma_{m}^{\prime \prime \prime}}\right)-i\left(K_{k}+K_{k}^{\prime}+K^{\prime \prime}\right)\left(\overline{\gamma_{l} \gamma_{k} \gamma_{i}^{\prime} \gamma_{j}^{\prime \prime} \gamma_{m}^{\prime \prime \prime}}\right)-i\left(K_{k}+K_{k}^{\prime}+K^{\prime \prime}\right)\left(\overline{\phi_{l} \phi_{k}^{\prime} \gamma_{i}^{\prime} \gamma_{j}^{\prime \prime} \gamma_{m}^{\prime \prime \prime}}\right) \\
& +i\left(K_{k}+K_{k}^{\prime}+K^{\prime \prime}\right)\left(\overline{\gamma_{l} \gamma_{k}^{\prime} \gamma_{i}^{\prime} \gamma_{j}^{\prime \prime} \gamma_{m}^{\prime \prime \prime}}\right)+i \frac{1}{\rho}\left(K_{k}+K_{k}^{\prime}+K^{\prime \prime}\right)\left(\overline{P \gamma_{i}^{\prime} \gamma_{j}^{\prime \prime} \gamma_{m}^{\prime \prime \prime}}\right)
\end{aligned}
$$

The tensor equation (14) can be converted to the scalar equation by contraction of the indices $\mathrm{i}$ and $\mathrm{j}$;

$$
\begin{aligned}
& \frac{\partial}{\partial t}\left(\overline{\phi_{l} \gamma_{i}^{\prime} \gamma_{i}^{\prime \prime} \gamma_{m}^{\prime \prime \prime}}\right)+\frac{v}{P_{r}}\left[( 1 + P _ { r } ) \left(K^{2}+\left(1+P_{r}\right) K^{\prime 2}+\left(1+P_{r}\right) K^{\prime \prime 2}+2 P_{r} K K^{\prime}+2 P_{r} K K^{\prime \prime}+\right.\right. \\
& \left.\left.2 P_{r} K K^{\prime \prime \prime}\right] \overline{\left(\phi_{l} \gamma_{i}^{\prime \prime} \gamma_{i}^{\prime \prime \prime}\right.}\right)-f\left(u_{i}-v_{i}\right) \overline{\phi_{l} \gamma_{i}^{\prime} \gamma_{i}^{\prime \prime} \gamma_{m}^{\prime \prime \prime}}=i\left(K_{k}+K_{k}^{\prime}+K^{\prime \prime}\right)\left(\overline{\phi_{l} \phi_{k} \gamma_{i}^{\prime \prime} \gamma_{i}^{\prime \prime} \gamma_{m}^{\prime \prime}}\right)-i\left(K_{k}+K_{k}^{\prime}+K^{\prime \prime}\right)\left(\overline{\gamma_{l} \gamma_{k} \gamma_{i}^{\prime} \gamma_{i}^{\prime \prime} \gamma_{m}^{\prime \prime}}\right)- \\
& i\left(K_{k}+K_{k}^{\prime}+K^{\prime \prime}\right)\left(\overline{\phi_{l} \phi_{k}^{\prime} \gamma_{i}^{\prime} \gamma_{i}^{\prime \prime} \gamma_{m}^{\prime \prime \prime}}\right)+i\left(K_{k}+K_{k}^{\prime}+K^{\prime \prime}\right)\left(\overline{\gamma_{l} \gamma_{k}^{\prime} \gamma_{i}^{\prime} \gamma_{i}^{\prime \prime} \gamma_{m}^{\prime \prime \prime}}\right)+i \frac{1}{\rho}\left(K_{k}+K_{k}^{\prime}+K^{\prime \prime}\right)\left(\overline{P \gamma_{i}^{\prime} \gamma_{i}^{\prime \prime} \gamma_{m}^{\prime \prime \prime}}\right)
\end{aligned}
$$


If we take the derivative with respect to $x_{i}$ of the momentum equation (1) at $\mathrm{p}$, we have

$$
\frac{\partial^{2} u_{j} u_{i}}{\partial x_{i} \partial x_{i}}=-\frac{1}{\rho} \frac{\partial^{2} P}{\partial x_{i} \partial x_{j}}
$$

Multiplying equation (16) by $T_{i}^{\prime} T_{j}^{\prime \prime} T_{m}^{\prime \prime \prime}$; and then taking time averages

$$
\frac{\partial^{2} \overline{\left(u_{j} u_{i} T_{i}^{\prime} T_{j}^{\prime \prime} T_{m}^{\prime \prime \prime}\right)}}{\partial x_{i} \partial x_{i}}=-\frac{1}{\rho} \frac{\partial^{2} \overline{\left(P T_{i}^{\prime} T_{j}^{\prime \prime} T_{m}^{\prime \prime \prime}\right)}}{\partial x_{i} \partial x_{j}}
$$

Equation (17) can be written in terms of the independent variables $\hat{r}, \hat{r}^{\prime}$ and $\hat{r}^{\prime \prime}$ we have,

$$
\begin{gathered}
{\left[\frac{\partial^{2}}{\partial r_{l} \partial r_{l}}+\frac{\partial^{2}}{\partial r_{l} \partial r_{l}^{\prime}}+\frac{\partial^{2}}{\partial r_{l}^{\prime \prime} \partial r_{l}^{\prime \prime}}+\frac{\partial^{2}}{\partial r_{l} \partial r_{l}^{\prime}}+\frac{\partial^{2}}{\partial r_{l} \partial r_{l}^{\prime \prime}}+\frac{\partial^{2}}{\partial r_{l} \partial r_{l}^{\prime \prime}}\right] \overline{\left(u_{j} u_{i} T_{i}^{\prime} T_{j}^{\prime \prime} T_{m}^{\prime \prime \prime}\right)}=} \\
-\frac{1}{\rho}\left[\frac{\partial^{2}}{\partial r_{l} \partial r_{k}}+\frac{\partial^{2}}{\partial r_{l} \partial r_{k}^{\prime}}+\frac{\partial^{2}}{\partial r_{l} \partial r_{k}^{\prime}}+\frac{\partial^{2}}{\partial r_{l}^{\prime} \partial r_{k}^{\prime \prime}}+\frac{\partial^{2}}{\partial r_{l}^{\prime} \partial r_{k}^{\prime \prime}}+\frac{\partial^{2}}{\partial r_{l} \partial r_{l}^{\prime \prime}}+\frac{\partial^{2}}{\partial r_{l}^{\prime} \partial r_{k}^{\prime}}+\frac{\partial^{2}}{\partial r_{l}^{\prime \prime} \partial r_{k}^{\prime \prime}}\right] \overline{\left(P T_{i}^{\prime} T_{j}^{\prime \prime} T_{m}^{\prime \prime \prime}\right)}
\end{gathered}
$$

and

$$
-\frac{1}{\rho} \overline{\left(P \gamma_{i}^{\prime} \gamma_{j}^{\prime \prime} \gamma_{k}^{\prime \prime \prime}\right)}=\frac{K_{l} K_{k}+K_{l} K_{k}^{\prime}+K_{l}^{\prime} K_{k}+K_{l}^{\prime} K_{k}^{\prime}+K_{l}^{\prime \prime} K_{k}+K_{l} K_{k}^{\prime \prime}+K_{l}^{\prime} K_{k}^{\prime \prime}+K_{l}^{\prime \prime} K_{k}^{\prime}+K_{l}^{\prime \prime} K_{k}^{\prime \prime}}{K_{l} K_{l}+K_{l}^{\prime} K_{l}^{\prime}+K_{l}^{\prime \prime} K_{l}^{\prime \prime}+2 K_{l} K_{l}^{\prime}+2 K_{l}^{\prime \prime} K_{l}+2 K_{l}^{\prime} K_{l}^{\prime \prime}} \overline{\phi_{l} \phi_{k} \gamma_{i}^{\prime} \gamma_{i}^{\prime \prime} \gamma_{m}^{\prime \prime \prime}}
$$

Equation (19) can be used to eliminate $\left(\overline{-\frac{1}{\rho} P \gamma_{i}^{\prime} \gamma_{j}^{\prime \prime} \gamma_{m}^{\prime \prime \prime}}\right)$ from equation (16) and (17). Taking contraction of the indices i and $\mathrm{j}$ in equation (19). Equations (16) and (19) are the spectral equation corresponding to the four -point correlation equation. The spectral equations corresponding to the three-point correlation equations by contraction of the indices $\mathrm{i}$ and $\mathrm{m}$ are

$$
\begin{aligned}
& \frac{\partial}{\partial t}\left(\overline{\phi_{i} \beta_{i}^{\prime} \beta_{i}^{\prime \prime}}\right)+\frac{v}{P_{r}}\left[\left(1+P_{r}\right)\left(K^{2}+K^{\prime 2}+2 P_{r} K K^{\prime}\right]\left(\overline{\phi_{i} \beta_{i}^{\prime} \beta_{i}^{\prime \prime}}\right)=i\left(K_{k}+K_{k}^{\prime}\right)\left(\overline{\phi_{i} \phi_{k} \beta_{i}^{\prime} \beta_{i}^{\prime \prime}}\right)-i\left(K_{k}+K_{k}^{\prime}\right)\left(\overline{\beta_{i} \beta_{k} \beta_{i}^{\prime} \beta_{i}^{\prime \prime}}\right)\right. \\
& -i\left(K_{k}+K_{k}^{\prime}\right)\left(\overline{\phi_{i} \phi_{k}^{\prime} \beta_{i}^{\prime} \beta_{i}^{\prime \prime}}\right)+i\left(K_{k}+K_{k}^{\prime}\right)\left(\overline{\phi_{i}^{\prime} \phi_{k} \beta_{i}^{\prime} \beta_{i}^{\prime \prime}}\right)+i\left(K_{k}+K_{k}^{\prime}\right)\left(\overline{\gamma \beta_{i}^{\prime} \beta_{i}^{\prime \prime}}\right)+f\left(u_{i}-v_{i}\right) \overline{\phi_{l} \gamma_{i}^{\prime} \gamma_{i}^{\prime \prime} \gamma_{i}^{\prime \prime \prime}}
\end{aligned}
$$

And

$$
-\frac{1}{\rho}\left(\overline{\gamma \beta_{i}^{\prime} \beta_{i}^{\prime \prime}}\right)=\frac{K_{i} K_{k}+K_{i} K_{k}^{\prime}+K_{i}^{\prime} K_{k}+K_{i}^{\prime} K_{k}^{\prime}}{K_{l}^{2}+K_{l}^{\prime 2}+2 K_{i} K_{i}^{\prime}}\left(\overline{\phi_{i} \phi_{k}^{\prime} \beta_{i}^{\prime} \beta_{i}^{\prime \prime}}\right)
$$

the six dimensional spectral tensors are defined by

$$
\begin{aligned}
\left\langle u_{i} T_{i}^{\prime} T_{j}^{\prime \prime}\left(\hat{r}^{\prime}\right)\right\rangle & =\int_{-\infty}^{\infty} \int_{-\infty}^{\infty}\left\langle\phi_{i} \beta_{i}^{\prime}(\hat{k}) \beta_{j}^{\prime \prime}(\hat{k})\right\rangle \exp \left[i\left(\hat{k} \cdot \hat{r}+\hat{k}^{\prime} \cdot \hat{r}^{\prime}\right) d \hat{k} d \hat{k}^{\prime}\right. \\
\left\langle u_{j} u_{i} T_{i}^{\prime} T_{j}^{\prime \prime}\left(\hat{r}^{\prime}\right)\right\rangle & =\int_{-\infty}^{\infty} \int_{-\infty}^{\infty}\left\langle\phi_{j} \phi_{i} \beta_{i}^{\prime}(\hat{k}) \beta_{j}^{\prime \prime}(\hat{k})\right\rangle \exp \left[i\left(\hat{k} \cdot \hat{r}+\hat{k}^{\prime} \cdot \hat{r}^{\prime}\right) d \hat{k} d \hat{k}^{\prime}\right. \\
\left\langle u_{i} u_{i} T_{i}^{\prime} T_{j}^{\prime \prime}\left(\hat{r}^{\prime}\right)\right\rangle & =\int_{-\infty}^{\infty} \int_{-\infty}^{\infty}\left\langle\phi_{i} \phi_{i} \beta_{i}^{\prime}(\hat{k}) \beta_{j}^{\prime \prime}(\hat{k})\right\rangle \exp \left[i\left(\hat{k} \cdot \hat{r}+\hat{k}^{\prime} \cdot \hat{r}^{\prime}\right) d \hat{k} d \hat{k}^{\prime}\right. \\
\left\langle u_{j} u_{i}^{\prime} T_{i}^{\prime}(\hat{r}) T_{j}^{\prime \prime}\left(\hat{r}^{\prime}\right)\right\rangle & =\int_{-\infty}^{\infty} \int_{-\infty}^{\infty}\left\langle\phi_{j} \phi_{i} \beta_{i}^{\prime}(\hat{k}) \beta_{j}^{\prime \prime}(\hat{k})\right\rangle \exp \left[i\left(\hat{k} \cdot \hat{r}+\hat{k}^{\prime} \cdot \hat{r}^{\prime}\right) d \hat{k} d \hat{k}^{\prime}\right. \\
\left\langle u_{j} u_{i}^{\prime \prime} T_{i}^{\prime}(\hat{r}) T_{j}^{\prime \prime}\left(\hat{r}^{\prime}\right)\right\rangle & =\int_{-\infty}^{\infty} \int_{-\infty}^{\infty}\left\langle\phi_{j} \phi_{i}^{\prime \prime} \beta_{i}^{\prime}(\hat{k}) \beta_{j}^{\prime \prime}(\hat{k})\right\rangle \exp \left[i\left(\hat{k} \cdot \hat{r}+\hat{k}^{\prime} \cdot \hat{r}^{\prime}\right) d \hat{k} d \hat{k}^{\prime}\right. \\
\left\langle P T_{i}^{\prime}(\hat{r}) T_{j}^{\prime \prime}\left(\hat{r}^{\prime}\right)\right\rangle & =\int_{-\infty}^{\infty} \int_{-\infty}^{\infty}\left\langle\gamma \beta_{i}^{\prime}(\hat{k}) \beta_{j}^{\prime \prime}(\hat{k})\right\rangle \exp \left[i\left(\hat{k} \cdot \hat{r}+\hat{k}^{\prime} \cdot \hat{r}^{\prime}\right) d \hat{k} d \hat{k}^{\prime}\right.
\end{aligned}
$$


Interchanging the points $\mathrm{P}^{\prime}$ and $\mathrm{P}^{\prime \prime}$ shows that

$$
\overline{\left\langle u_{j} u_{i}^{\prime \prime} T^{\prime \prime} T^{\prime}\right\rangle}=\overline{\left\langle u_{j} u_{i}^{\prime} T^{\prime} T^{\prime \prime}\right\rangle}=\int_{-\infty}^{\infty} \int_{-\infty}^{\infty} \overline{\beta_{j} \beta_{i}^{\prime} \theta^{\prime} \theta^{\prime \prime}} \exp \left[i\left(\hat{K} \cdot \hat{r}+\hat{K}^{\prime} \cdot \hat{r}^{\prime}\right)\right] d \hat{K} d \hat{K}
$$

The spectral equations corresponding to the two-point correlation equations by contraction of the indices $i$ and $j$ are

$$
\frac{\partial}{\partial t}\left(\overline{\phi_{i} \beta_{i}^{\prime}}\right)+\frac{2 v}{P_{r}} K^{2}\left(\overline{\phi_{i} \beta_{i}^{\prime}}\right)=2 i K_{k}\left[\left(\overline{\phi_{i} \phi_{k}^{\prime} \beta_{i}^{\prime}}\right)-\left(\overline{\phi_{i}^{\prime} \phi_{k} \beta_{i}^{\prime}}\right)\right.
$$

A relation between $\phi_{i} \phi_{k}^{\prime} \beta_{i}^{\prime}(\hat{k})$ and $\phi_{i}^{\prime} \phi_{k} \beta_{i}^{\prime}(\hat{k})$ can be obtained by letting $\hat{r}^{\prime \prime}=0$ in equation (7) and comparing the result with equation (23)

$$
\begin{aligned}
\left\langle u_{i}^{\prime} T_{i}^{\prime}(\hat{r})\right\rangle & =\int_{-\infty}^{\infty}\left\langle\phi_{i}^{\prime} \beta_{i}^{\prime}(\hat{k})\right\rangle \exp [i(\hat{k} . \hat{r})] d \hat{k} \\
\left\langle u_{i} u_{k}^{\prime} T_{i}^{\prime}(\hat{r})\right\rangle & =\int_{-\infty}^{\infty}\left\langle\phi_{i} \phi_{k}^{\prime} \beta_{i}^{\prime}(\hat{k})\right\rangle \exp [i(\hat{k} . \hat{r})] d \hat{k} \\
\left\langle u_{i}^{\prime} u_{k} T_{i}^{\prime}(\hat{r})\right\rangle & =\int_{-\infty}^{\infty}\left\langle\phi_{i}^{\prime} \phi_{k} \beta_{i}^{\prime}(\hat{k})\right\rangle \exp [i(\hat{k} . \hat{r})] d \hat{k}
\end{aligned}
$$

\section{Solution}

To obtain the equation for the timesbe fore thefinal period of decay, the four point correlations are considered and the quintuple correlation terms decays faster than the lower order correlation terms. If this assumption is made than we can neglect all the terms on the right side of the equation (15).

So we get

$$
\frac{\partial}{\partial t}\left(\overline{\phi_{l} \gamma_{i}^{\prime} \gamma_{i}^{\prime \prime} \gamma_{m}^{\prime \prime \prime}}\right)+\frac{v}{P_{r}}\left[\left(1+P_{r}\right)\left(K^{2}+\left(1+P_{r}\right) K^{\prime 2}+\left(1+P_{r}\right) K^{\prime \prime 2}+2 P_{r} K K^{\prime}+2 P_{r} K K^{\prime \prime}+2 P_{r} K K^{\prime \prime \prime}\right] \overline{\left(\phi_{l} \gamma_{i}^{\prime} \gamma_{i}^{\prime \prime} \gamma_{m}^{\prime \prime \prime}\right.}\right)-f\left(u_{i}-v_{i}\right) \overline{\phi_{i} \gamma_{i}^{\prime} \gamma_{i}^{\prime \prime} \gamma_{m}^{\prime \prime \prime}}=0
$$

Integrating this equation between $\mathrm{t}_{1}$ and $\mathrm{t}$ with inner multiplication by $k_{k}$ we have,

$$
\left\langle\phi_{l} \gamma_{i}^{\prime} \gamma_{j}^{\prime \prime} \gamma_{m}^{\prime \prime}\right\rangle=\left\langle\phi_{l} \gamma_{i}^{\prime} \gamma_{j}^{\prime \prime} \gamma_{m}^{\prime \prime \prime}\right\rangle_{1} \exp \left\{\frac{v}{P_{r}}\left[\left(1+P_{r}\right)\left(K^{2}+\left(1+P_{r}\right) K^{\prime 2}+\left(1+P_{r}\right) K^{\prime \prime 2}+2 P_{r} K K^{\prime}+2 P_{r} K K^{\prime \prime}+2 P_{r} K K^{\prime \prime \prime}\right]+f\left(u_{i}-v_{i}\right)\right\}\left(t-t_{1}\right)\right.
$$

Where $\left\langle\phi_{l} \gamma_{i}^{\prime} \gamma_{j}^{\prime \prime} \gamma_{m}^{\prime \prime \prime}\right\rangle_{1}$ is the value of $\left\langle\phi_{l} \gamma_{i}^{\prime} \gamma_{j}^{\prime \prime} \gamma_{m}^{\prime \prime \prime}\right\rangle$ at $\mathrm{t}=\mathrm{t}_{1}$ for small values of $\mathrm{k}$,

Substituting of equation (21), (28), (33) in equation (20), we get,

$$
\begin{aligned}
& \frac{\partial}{\partial t}\left(k_{k} \overline{\phi_{i} \beta_{i}^{\prime} \beta_{i}^{\prime \prime}}\right)+\frac{v}{P_{r}}\left[\left(1+P_{r}\right)\left(K^{2}+K^{\prime 2}+2 P_{r} K K^{\prime}\right]\left(k_{k} \overline{\phi_{i} \beta_{i}^{\prime} \beta_{i}^{\prime \prime}}\right)=\right. \\
& {[a]_{1} \int_{-\infty}^{\infty} \exp \left[-\frac{v}{P_{r}}\left(t-t_{1}\right)\left[\left(1+P_{r}\right)\left(k^{2}+k^{\prime 2}+k^{\prime \prime 2}\right)+2 p_{r}\left(k k^{\prime}+k^{\prime} k^{\prime \prime}+k^{\prime \prime} k\right)\right\}\right] \exp \left[f_{S}\left(t-t_{1}\right)\right] d k^{\prime \prime}+} \\
& {[b]_{1} \int_{-\infty}^{\infty} \exp \left[-\frac{v}{P_{r}}\left(t-t_{1}\right)\left[\left(1+P_{r}\right)\left(k^{2}+k^{2}+k^{\prime \prime 2}\right)+2 p_{r} k k^{\prime}-2 p_{r} k^{\prime \prime} k\right)\right\} \exp \left[f_{S}\left(t-t_{1}\right)\right] d k^{\prime \prime}+} \\
& {[c]_{1} \int_{-\infty}^{\infty} \exp \left[-\frac{v}{P_{r}}\left(t-t_{1}\right)\left[\left(1+P_{r}\right)\left(k^{2}+k^{\prime 2}+k^{\prime \prime 2}\right)+2 p_{r} k k^{\prime}-2 p_{r} k^{\prime \prime} k^{\prime}\right)\right\} \exp \left[f_{S}\left(t-t_{1}\right)\right] d k^{\prime \prime}}
\end{aligned}
$$

At $t_{1}$, the functions $\gamma$ shave been assumed independent that assumption is not, made for other times. This is one of several assumptions made concerning the initial conditions, although continuity equation satisfied the conditions. The complete specification of initial condition is difficult; the assumptions for the initial conditions made here. Substituting $d k^{\prime \prime}=d k_{1}^{\prime \prime} d k_{2}^{\prime \prime} d k_{3}^{\prime \prime}$ and integrating equation (34) with respect to $k_{1}^{\prime \prime}, k_{2}^{\prime \prime}$ and $k_{3}^{\prime \prime}$, 
We get,

$$
\begin{gathered}
\frac{\partial}{\partial t}\left(k_{k} \overline{\varphi_{i} \beta_{i}^{\prime} \beta_{i}^{\prime \prime}}\right)+\frac{v}{P_{r}}\left[\left(1+P_{r}\right)\left(K^{2}+K^{\prime 2}\right)+2 P_{r} K K^{\prime}\right]\left(k_{k} \overline{\varphi_{i} \beta_{i}^{\prime} \beta_{i}^{\prime \prime}}\right)=\left(\frac{\pi p_{r}}{v\left(t-t_{1}\right)\left(1+p_{r}\right)}\right)^{\frac{1}{2}} \\
{\left[\mathrm{a}_{1}\right] \exp \left[-\frac{v\left(t-t_{1}\right)\left[\left(1+P_{r}\right)\right.}{P_{r}}\left\{\frac{\left(1+2 P_{r}\right)\left(k^{2}+k^{\prime 2}\right)}{\left(1+P_{r}\right)^{2}}+\frac{2 P_{r} k k^{\prime}}{\left(1+P_{r}\right)^{2}}\right\}\right] \exp \left[f_{s}\left(t-t_{1}\right)\right]+\left(\frac{\pi p_{r}}{v\left(t-t_{1}\right)\left(1+p_{r}\right)}\right)^{\frac{1}{2}}} \\
{\left[\mathrm{~b}_{1}\right] \exp \left[-\frac{v\left(t-t_{1}\right)\left[\left(1+P_{r}\right)\right.}{P_{r}}\left\{\frac{\left(1+2 P_{r}\right) k^{2}}{\left(1+P_{r}\right)^{2}}+\frac{2 P_{r} k k^{\prime}}{\left(1+P_{r}\right)}+k^{\prime 2}\right\}\right] \exp \left[f_{S}\left(t-t_{1}\right)\right]+\left(\frac{\pi p_{r}}{v\left(t-t_{1}\right)\left(1+p_{r}\right)}\right)^{\frac{1}{2}}} \\
{\left[c_{1}\right] \exp \left[-\frac{v\left(t-t_{1}\right)\left[\left(1+P_{r}\right)\right.}{P_{r}}\left\{k^{2}+\frac{\left(1+2 P_{r}\right)\left(k^{\prime 2}\right)}{\left(1+P_{r}\right)^{2}}+\frac{2 P_{r} k k^{\prime}}{\left(1+P_{r}\right)}\right\}\right] \exp \left[f_{S}\left(t-t_{1}\right)\right]}
\end{gathered}
$$

Integrating equation (35) with respect to time, and in order to simplify the calculations, we will assume that $[a]_{1}=0$; Substituting of equation (32) in equation (29) and setting $T=2 \pi k^{2} \varphi_{i} \varphi_{i}^{\prime}$, result in

$$
\frac{\partial T}{\partial t}+\frac{2 v k^{2}}{p_{r}} T=W
$$

Where,

$$
\begin{aligned}
& W=k^{2} \int_{-\infty}^{\infty} 2 \pi . i\left[\left\langle k_{k} \phi_{l} \beta_{i}^{\prime} \beta_{i}^{\prime \prime}\left(\hat{k}, \hat{k}^{\prime}\right)\right\rangle-\left\langle k_{k} \phi_{l} \beta_{i}^{\prime} \beta_{i}^{\prime \prime}\left(-\hat{k},-\hat{k}^{\prime}\right)\right\rangle\right]_{0} \exp \left[-\frac{v}{P_{r}}\left(t-t_{1}\right)\left\{\left(1+P_{r}\right)\left(k^{2}+k^{2}\right)+2 p_{r} k k^{\prime}\right\} d k^{\prime}\right] \\
& +k^{2} \int_{-\infty}^{\infty} \frac{2 p_{r} \cdot \pi^{\frac{5}{2}}}{v} i\left[b\left(\hat{k} \cdot \hat{k}^{\prime}\right)+b\left(\hat{k}-\hat{k}^{\prime}\right)\right]_{1} \exp \left[f_{s}\left(t-t_{1}\right)\right]+\left[k \exp \left[\left(-\omega^{2}\right)\left(1+p_{r}\right)\left(k^{2}+k^{2}\right)+2 p_{r} k k^{\prime}\right]\right]_{0}^{\omega k^{\prime}} / 2\left\{\exp \left(x^{2}\right) d x\right\} d k^{\prime} \\
& +(\operatorname{expk}) \times \exp \left[\left(-\omega^{2}\right)\left(\left(1+p_{r}\right)\left(k^{2}+k^{\prime 2}\right)+2 p_{r} k k^{\prime}\right)\right] \int_{0}^{\omega k^{\prime}} / 2\left\{\exp \left(x^{2}\right) d x\right\} d k^{\prime}
\end{aligned}
$$

$\mathrm{T}$ is the temperature fluctuations spectrum function, which represent contributions from various wave numbers to the energy and $\mathrm{W}$ is the energy transfer function, which is responsible for the transfer of energy between wave numbers. In order to make further calculations, an assumption must be made for the forms of the bracketed quantities with the subscripts 0 and 1 in equation (37) which depends on the initial conditions.

$$
(2 \pi)^{2}\left[\left\langle k_{k} \varphi_{l} \beta_{i}^{\prime} \beta_{i}^{\prime \prime}\left(\hat{k}, \hat{k}^{\prime}\right)\right\rangle-\left\langle k_{k} \varphi_{l} \beta_{i}^{\prime} \beta_{i}^{\prime \prime}\left(-\hat{k},-\hat{k}^{\prime}\right)\right\rangle\right]_{0}-\xi_{0}\left(k^{4} k^{\prime 6}-k^{6} k^{\prime 4}\right)
$$

where, $\xi_{0}$ is a constant depending on the initial conditions. For the other bracketed quantities in equation (37),

$$
\frac{4 p_{r} \cdot \pi^{\frac{7}{2}}}{v} i\left[b\left(\hat{k} \cdot \hat{k}^{\prime}\right)+b\left(\hat{k}+\hat{k}^{\prime}\right)\right]=\frac{4 p_{r} \cdot \pi^{\frac{7}{2}}}{v} i\left[c\left(\hat{k} \cdot \hat{k}^{\prime}\right)+c\left(\hat{k}+\hat{k}^{\prime}\right)\right]=-2 \xi_{1}\left(k^{6} k^{\prime 8}-k^{8} k^{\prime 6}\right)
$$

Remembering that $d \hat{k}^{\prime}=-2 \pi \cdot \hat{k}^{\prime 2} d(\cos \theta)$ and $k k^{\prime}=k k^{\prime} \cos \theta, \theta$ is the angle between $\hat{k}$ and $\hat{k}^{\prime}$ and carrying out the integration with respect to $\theta$, we get,

$$
\begin{aligned}
& \mathrm{W}=-\int_{0}^{\infty}\left[\frac { \xi _ { 0 } ( k ^ { 2 } k ^ { \prime 4 } - k ^ { 4 } k ^ { \prime 2 } ) k k ^ { \prime } } { v ( t - t _ { 0 } ) } \left\{\exp \left[-\frac{v}{P_{r}}\left(t-t_{0}\right)\left\{\left(1+P_{r}\right)\left(k^{2}+k^{\prime 2}\right)-2 p_{r} k k^{\prime}\right\}\right]-\right.\right. \\
& \left.\exp \left[-\frac{v}{P_{r}}\left(t-t_{0}\right)\left\{\left(1+P_{r}\right)\left(k^{2}+k^{\prime 2}\right)+2 p_{r} k k^{\prime}\right\}\right]\right\}+\frac{\xi_{1}\left(k^{4} k^{\prime 6}-k^{6} k^{\prime 4}\right) k k^{\prime}}{v\left(t-t_{0}\right)}+
\end{aligned}
$$




$$
\begin{aligned}
& \left.+k \cdot \exp \left[\left(-\omega^{2}\right)\left(\left(1+p_{r}\right)\left(k^{2}+k^{\prime 2}\right)-2 p_{r} k k^{\prime}\right)\right]-\mathrm{k} \cdot \exp \left[\left(-\omega^{2}\right)\left(\left(1+p_{r}\right)\left(k^{2}+k^{2}\right)+2 p_{r} k k^{\prime}\right)\right]\right\} \int_{0}^{\frac{\omega k}{2}} \exp \left(x^{2}\right) d x+ \\
& \left.\left\{k^{\prime} \exp \left[-\omega^{2}\left(\left(1+p_{r}\right)\left(k^{2}+k^{\prime 2}\right)-2 p_{r} k k^{\prime}\right)\right]-k^{\prime} \exp \left[-\omega^{2}\left(\left(1+p_{r}\right)\left(k^{2}+k^{2}\right)+2 p_{r} k k^{\prime}\right)\right]\right\} \int_{0}^{\frac{\omega k}{2}} \exp \left(x^{2}\right) d x\right] d k^{\prime}
\end{aligned}
$$

Where, $\omega=\left(\frac{v\left(t-t_{1}\right)\left[\left(1+P_{r}\right)\right.}{P_{r}}\right)^{\frac{1}{2}}$

By integrating equation (40) with respect to $\mathrm{k}^{\prime}$ We have,

$$
W=W_{\beta}+W_{\gamma} \exp \left[f_{s}\left(t-t_{1}\right)\right]
$$

Where,

$$
\begin{aligned}
& W_{\beta}=\frac{\pi^{\frac{1}{2}} \xi_{0} p_{r}}{v^{\frac{1}{2}}\left(t-t_{0}\right)^{\frac{5}{2}}\left(1+p_{r}\right)^{\frac{7}{2}}} \exp \left\{\frac{v\left(t-t_{0}\right)\left(1+p_{r}\right) k^{2}}{2 p_{r}\left(1+p_{r}\right)}\right\}\left[\frac{5 p_{r} k^{4}}{4 v^{2}\left(t-t_{0}\right)^{2}\left(1+p_{r}\right)}+\right. \\
& \left.\left\{\frac{5 p_{r}{ }^{2}}{\left(1+p_{r}\right)^{2} v\left(t-t_{0}\right)}-\frac{3}{2 v\left(t-t_{0}\right)}\right\} k^{6}+\frac{p_{r}}{\left(1+2 p_{r}\right)}\left\{\frac{1+p_{r}{ }^{2}}{\left(1+p_{r}\right)^{2}}\right\} k^{8}\right]
\end{aligned}
$$

And

$$
\begin{aligned}
& W_{r}=\frac{\pi^{\frac{1}{2}} \xi_{1} p_{r}{ }^{5}}{8 v^{2}\left(t-t_{1}\right)^{\frac{3}{2}}\left(1+p_{r}\right)^{5}} \exp \left\{\frac{-v\left(t-t_{1}\right)\left(1+2 p_{r}-p_{r}{ }^{2}\right)}{p_{r}\left(1+p_{r}\right)}\right\} k^{2}\left[\frac{90 p_{r} k^{6}}{v^{4}\left(t-t_{1}\right)^{4}\left(1+p_{r}\right)}+\right. \\
& 3\left\{\frac{4 p_{r}{ }^{2}}{\left(1+p_{r}\right) v^{2}\left(t-t_{1}\right)^{2}}+\frac{1}{\left(1+p_{r}\right)^{2} v^{3}\left(t-t_{1}\right)^{3}}-\frac{1}{v^{3}\left(t-t_{1}\right)^{3}} k^{8}+\right. \\
& \left.\left\{\frac{64 p_{r}{ }^{2}}{\left(1+p_{r}\right)^{2} v\left(t-t_{1}\right)}+\frac{10 p_{r}{ }^{3}}{\left(1+p_{r}\right)^{3} v^{2}\left(t-t_{1}\right)^{2}}-\frac{40}{v\left(t-t_{1}\right)}\right\} k^{10}+8\left\{\frac{p_{r}{ }^{2}}{\left(1+p_{r}\right)^{2}}+\frac{p_{r}}{\left(1+p_{r}\right)}\right\} k^{12}\right]+ \\
& \left.\left\{\frac{64 p_{r}{ }^{2}\left(1+p_{r}\right)^{2}}{\left(1+2 p_{r}\right)^{2} v\left(t-t_{1}\right)}+\frac{10 p_{r}{ }^{3}\left(1+p_{r}\right)^{3}}{\left(1+2 p_{r}\right)^{3} v^{2}\left(t-t_{1}\right)^{2}}-\frac{40}{v\left(t-t_{1}\right)}\right\} k^{10}+\left\{\frac{8 p_{r}{ }^{3}\left(1+p_{r}\right)^{3}}{\left(1+2 p_{r}\right)^{2}}-\frac{p_{r}\left(1+p_{r}\right)}{\left(1+2 p_{r}\right)}\right\} k^{12}\right]+ \\
& \frac{\pi^{\frac{1}{2}} \xi_{1} p_{r}{ }^{\frac{9}{2}}}{8 v^{\frac{3}{2}}\left(t-t_{1}\right)^{2}\left(1+2 p_{r}\right)^{\frac{9}{2}}} \exp \left\{\frac{-v\left(t-t_{1}\right)\left(1+2 p_{r}\right)}{p_{r}}\right\} k^{2} \cdot\left[\frac{90 p_{r} k^{7}}{v^{4}\left(t-t_{1}\right)^{4}\left(1+p_{r}\right)^{2}}+\right. \\
& \left\{\frac{120 p_{r}}{v^{2}\left(t-t_{1}\right)^{2}}+\frac{60 p_{r}{ }^{2}}{\left(1+p_{r}\right)^{2} v^{3}\left(t-t_{1}\right)^{3}}-\frac{1}{v^{3}\left(t-t_{1}\right)^{3}}\right\} k^{9}+ \\
& \left\{\frac{64 p_{r}{ }^{2}}{v\left(t-t_{1}\right)}+\frac{60 p_{r}{ }^{3}}{v^{2}\left(t-t_{1}\right)^{2}\left(1+p_{r}\right)^{2}}-\frac{40\left(1+p_{r}\right)^{2}}{v\left(t-t_{1}\right)}\right\} k^{11}+\left\{p_{r}{ }^{2}-p_{r}\left(1+p_{r}\right)^{2}\right\} \int_{0}^{2} \exp \left(y^{2}\right) d y
\end{aligned}
$$

Where, $\omega_{1}=\left(\frac{v\left(t-t_{1}\right)\left[\left(1+P_{r}\right)\right.}{P_{r}}\right)^{\frac{1}{2}} k$

The integral expression in equation (41),The quantity $W_{\beta}$ is the energy transfer function arising from consideration of Temperature fluctuation field at three point correlation equation; $W_{\gamma}$ arises from consideration of the four -point correlation equation. Integration of equation (41) over all wave numbers shows that

$$
\int_{0}^{\infty} W \cdot d \vec{k}=0
$$


The expression for $\mathrm{W}$ satisfies the conditions of continuity and homogeneity, physically, it was to be expected, Since W is a measure of transfer of energy and the numbers must be zero. From (36), we get,

$$
\begin{aligned}
T & =\exp \left[-\frac{2 v k^{2}\left(t-t_{0}\right)}{p_{r}}\right] \int W \exp \left[-\frac{2 v k^{2}\left(t-t_{0}\right)}{p_{r}}\right] d t+J(k) \exp \left[-\frac{2 v k^{2}\left(t-t_{0}\right)}{p_{r}}\right] \\
& =J(k) \exp \left[-\frac{2 v k^{2}\left(t-t_{0}\right)}{p_{r}}\right]+\exp \left[-\frac{2 v k^{2}\left(t-t_{0}\right)}{p_{r}}\right] \int\left(W_{\beta}+W_{\gamma}\right) \exp \left[-\frac{2 v k^{2}\left(t-t_{0}\right)}{p_{r}}\right] d t
\end{aligned}
$$

Where, $J(k)=\frac{N_{0} k^{2}}{\pi}$ is a constant of integration and can be obtained as by Corrsin $\mathrm{S}$

Where,

$$
W=W_{\beta}+W_{\gamma}
$$

After integration of equation (43) becomes

$$
T=J(k) \exp \left[-\frac{2 v k^{2}\left(t-t_{0}\right)}{p_{r}}\right]+T_{\beta}+T_{\gamma}
$$

Where,

$$
\begin{aligned}
& T_{\beta}=\frac{\pi^{\frac{1}{2}} \xi_{0} p_{r}{ }^{\frac{5}{2}}}{8 v^{\frac{3}{2}}\left(1+p_{r}\right)^{\frac{7}{2}}} \exp \left\{-\frac{v\left(t-t_{0}\right)\left(1+2 p_{r}\right) k^{2}}{p_{r}\left(1+p_{r}\right)}\right\}+\left[\frac{3 p_{r} k^{4}}{\left.2 v^{2}\left(t-t_{0}\right)^{\frac{5}{2}}\right)}+\right. \\
& \left.\left\{\frac{5 p_{r}{ }^{2}-6 p_{r}}{3 v\left(1+p_{r}\right)\left(t-t_{0}\right)^{\frac{3}{2}}}\right\} k^{6}-\left\{\frac{3 p_{r}{ }^{2}-2 p_{r}+3}{3\left(1+p_{r}{ }^{2}\right)\left(t-t_{0}\right)^{\frac{1}{2}}}\right\} k^{8}+\left\{\frac{8 v^{\frac{1}{2}}\left(3 p_{r}{ }^{2}-2 p_{r}+3\right.}{3\left(1+p_{r}\right)^{\frac{5}{2}} p_{r}{ }^{\frac{1}{2}}}\right\} k^{9} F(\omega)\right]
\end{aligned}
$$

Where,

$$
F(\omega)=\exp \left(-\omega^{2}\right) \int_{0}^{\omega} \exp \left(x^{2}\right) d x \quad, \omega=\left(\frac{v\left(t-t_{0}\right)}{P_{r}\left(1+P_{r}\right)}\right)^{\frac{1}{2}} k
$$

And,

$$
\begin{aligned}
& T_{\gamma}=\frac{\pi^{\frac{1}{2}} \xi_{1} p_{r}{ }^{5}}{8 v^{2}\left(1+p_{r}\right)^{5}} \exp \left\{\frac{-v\left(t-t_{1}\right)\left(1+2 p_{r}-p_{r}{ }^{2}\right)}{p_{r}\left(1+p_{r}\right)}\right\} k^{2}\left[\frac{18 p_{r} k^{6}}{v^{4}\left(t-t_{1}\right)^{5}\left(1+p_{r}\right)}+\right. \\
& \left\{\frac{15-6 p_{r}+21 p_{r}{ }^{2}}{4\left(1+p_{r}\right)^{2} v^{3}\left(t-t_{1}\right)^{4}}+\frac{4 p_{r}}{\left(1+p_{r}\right) v^{2}\left(t-t_{1}\right)^{3}}-\frac{1}{v^{3}\left(t-t_{1}\right)^{3}}\right\} k^{8}+ \\
& \left\{\frac{15-6 p_{r}+36 p_{r}{ }^{2}-6 p_{r}{ }^{4}}{12\left(1+p_{r}\right)^{3} v^{2}\left(t-t_{1}\right)^{3}}+\frac{14 p_{r}{ }^{2}-404 p_{r}-18}{\left(1+p_{r}\right)^{2} v\left(t-t_{1}\right)}\right\} k^{10} \cdot \exp \left(-\omega_{1}\right) \int E i\left(\omega_{1}\right) d t+ \\
& \frac{\left(-\pi^{\frac{1}{2}} \xi_{1} p_{r}{ }^{\frac{9}{2}}\right.}{128 v^{2}\left(1+p_{r}\right)^{\frac{11}{2}}} \exp \left\{\frac{-v\left(t-t_{1}\right)\left(1+2 p_{r}\right)}{p_{r}}\right\} k^{2}\left[\frac{180 p_{r}\left(1+2 p_{r}\right)}{v^{4}\left(t-t_{1}\right)^{4}\left(1+2 p_{r}\right)^{6}}\right] k^{6}+ \\
& \left\{\frac{1710-1036 p_{r}-1820 p_{r}{ }^{2}}{4\left(1+p_{r}\right)^{2} v^{3}\left(t-t_{1}\right)^{4}}+\frac{140 p_{r}\left(1+p_{r}\right)}{3\left(1+2 p_{r}\right) v^{2}\left(t-t_{1}\right)^{3}}-\frac{1}{v^{3}\left(t-t_{1}\right)^{3}}\right\} k^{8}+
\end{aligned}
$$

Where,

$$
E i\left(\omega_{1}\right)=\frac{\exp \left\{\frac{-\left(1+2 p_{r}{ }^{2}\right) t k^{2}}{p_{r}\left(1+p_{r}\right)}\right\}}{t-t_{1}} \quad \text { and }, \omega_{1}=\frac{-\left(1+2 p_{r}{ }^{2}\right) t k^{2}}{p_{r}\left(1+p_{r}\right)}
$$

From equation (45),

$$
T=T_{1}+T_{2}
$$


Where, $T_{1}=J(k) \exp \left[-\frac{2 v k^{2}\left(t-t_{0}\right)}{p_{r}}\right]+T_{\beta} \quad$ and $\quad T_{2}=T_{\gamma}$

In equation (48) $T_{1}$ and $T_{2}$ are temperature fluctuation field spectrum arising from consideration of the three and four -point correlation equations respectively. Equation (48) can be integrated over of all wave numbers to give the total Temperature fluctuation turbulent energy. That is

$$
\begin{gathered}
\left\langle\frac{T \cdot T^{\prime}}{2}\right\rangle=\int_{0}^{\infty} T d k=\int_{0}^{\infty}\left(T_{1}+T_{2}\right) d k \\
\text { Now, } \int_{0}^{\infty} T_{1} d k=\frac{N_{0} p_{r}^{\frac{3}{2}} v^{-\frac{3}{2}}\left(t-t_{0}\right)^{-\frac{5}{2}}}{32 \sqrt{2 \pi}}+\xi_{0} B_{0} v^{-5}\left(t-t_{0}\right)^{-7}
\end{gathered}
$$

And

$$
\int_{0}^{\infty} T_{2} d k=\xi_{1}\left[R v^{-\frac{13}{2}}\left(t-t_{1}\right)^{-\frac{11}{2}}+S v^{-\frac{15}{2}}\left(t-t_{1}\right)^{-\frac{13}{2}}\right] \exp \left[f S\left(t-t_{1}\right)\right] .
$$

Where, $\mathrm{R}=\mathrm{C}_{2}+\mathrm{C}_{4}+\mathrm{C}_{6}+\ldots$ And $\mathrm{S}=\mathrm{C}_{1}+\mathrm{C}_{3}+\mathrm{C}_{5}+\ldots$

$$
\begin{aligned}
& B_{0}=\frac{\pi p_{r}{ }^{6}}{\left(1+p_{r}\right)\left(1+2 p_{r}\right)^{\frac{5}{2}}}\left[\frac{8}{13}+\frac{3 p_{r}\left(1+5 p_{r}\right)}{2\left(1+2 p_{r}\right)}+\ldots . .\right] \\
& C_{1}=\frac{\pi p_{r} \frac{7}{2}}{\left(1+p_{r}\right)^{\frac{5}{2}}\left(1+2 p_{r}-P_{m}{ }^{2}\right)^{\frac{7}{2}}}\left[\frac{13.7}{32}+\frac{13.5\left(13-4 p_{r}+15 p^{2}{ }^{2}\right)}{2\left(1+2 p_{r}\right)}+\ldots . .\right] \\
& C_{2}=\frac{\pi p_{r} \frac{19}{2}}{\left(1+p_{r}\right)^{\frac{3}{2}}\left(1+2 p_{r}-P_{r}{ }^{2}\right)^{\frac{5}{2}}}\left[\frac{11.7}{32}+\frac{13.7 .5\left(15 p_{r}-14+20 p^{2} r\right)}{2^{7}\left(1+2 p_{r}-P_{r}{ }^{2}\right)}+\ldots . .\right] \\
& C_{3}=\frac{\pi p_{r} \frac{17}{2}\left(1+p_{r}\right)^{\frac{1}{2}}}{\left(1+p_{r}\right)^{2}\left(1+2 p_{r}-P_{r}{ }^{2}\right)^{\frac{5}{2}}}\left[\frac{13.7}{32}+\frac{13.5\left(15+28 p_{r}-14 P^{2}{ }_{r}+20 p^{4}{ }_{r}\right)}{2^{9}\left(1+p_{r}\right)^{2}\left(1+2 p_{r}-P_{r}{ }^{2}\right)}+\ldots . .\right] \\
& C_{4}=\frac{\pi p_{r} \frac{21}{2}}{\left(1+p_{r}\right)^{\frac{3}{2}}\left(1+2 p_{r}-P_{m}{ }^{2}\right)^{\frac{7}{2}}}\left[\frac{11.7 .3}{16}+\frac{13.7 .5\left(-20 p_{r}-17 P_{r}^{2}+20 p_{r}{ }^{3}\right)}{2^{7}\left(1+2 p_{r}-P_{m}{ }^{2}\right)}+\ldots . .\right] \\
& C_{5}=\frac{\pi p_{r} \frac{17}{2}\left(1+p_{r}\right)^{\frac{1}{2}}}{\left(1+p_{r}\right)^{\frac{17}{2}}\left(1+2 p_{r}\right)^{\frac{7}{2}}}\left[\frac{13.7 .5 .3}{32}-\frac{13.5 .3\left(15+28 p_{r}-14 P^{3}{ }_{r}+20 p^{5}{ }_{r}\right)}{2^{11}\left(1+p_{r}\right)^{2}}+\ldots . .\right] \\
& C_{6}=\frac{\pi p_{r}{ }^{\frac{21}{2}}}{\left(1+p_{r}\right)^{\frac{11}{2}}\left(1+2 p_{r}\right)^{\frac{9}{2}}}\left[\frac{13.11 .7 .3}{16}-\frac{11.9 .7 .5\left(20+20 p_{r}-17 P_{r}{ }^{2}+20 p_{r}{ }^{3}\right)}{2^{7}\left(1+2 p_{r}-P_{m}{ }^{2}\right)}+\ldots . .\right] \\
& \frac{\left\langle T \cdot T^{\prime}\right\rangle}{2}=\frac{N_{0} p^{\frac{3}{2}} r v^{-\frac{3}{2}}\left(t-t_{0}\right)^{-\frac{3}{2}}}{6 \sqrt{2 \pi}}+\xi_{0} X v^{-5}\left(t-t_{0}\right)^{-5} \exp \left[f_{S}\left(t-t_{1}\right)\right]+\xi_{1}\left[R v^{-\frac{13}{2}}\left(t-t_{1}\right)^{-\frac{11}{2}}+S v^{-\frac{15}{2}}\left(t-t_{1}\right)^{-\frac{13}{2}}\right] \cdot \exp \left[f_{S}\left(t-t_{1}\right)\right]
\end{aligned}
$$

Also, we can write equation (50) of the following form, 


$$
\left\langle T^{2}\right\rangle=W\left(t-t_{0}\right)^{-3 / 2}+X\left(t-t_{0}\right)^{-5} \exp \left[l\left(t-t_{1}\right)\right]+Y\left(t-t_{1}\right)^{-\frac{7}{2}} \exp \left[l\left(t-t_{1}\right)\right]+Z\left(t-t_{1}\right)^{-7} \exp \left[l\left(t-t_{1}\right)\right]
$$

This is the decay of energy of Temperature fluctuation in dusty fluid homogeneous turbulence for four point correlations.

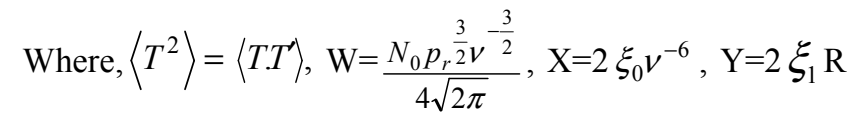
$v^{-\frac{9}{2}}$ and $\mathrm{Z}=2 \xi_{1} S v^{-\frac{11}{2}}$,

$l=f_{S}$ the dust particle parameters

If $\mathrm{R}=0$ and $\mathrm{S}=0$ that is $Y=0$ and $Z=0$ in equation (53), it will be in the form

$$
\begin{gathered}
\left\langle T^{2}\right\rangle=A\left(t-t_{0}\right)^{-3 / 2}+B\left(t-t_{0}\right)^{-5} \exp \left[f_{s}\left(t-t_{1}\right)\right], \text { Taking } \\
W=A, X=B
\end{gathered}
$$

Which is completely same with the result of Azad and Sarker (2009) for the case of three -point correlation for dusty fluid. In equation (54), the third and fourth term on the right hand side comes due to four point correlations for dusty fluid.

\section{Result and Discussions}

The evaluation of analytical results reported in this paper is performed and representative set of results is reported graphically. These results are obtained to illustrate the influence of various parameters on the temperature fluctuations and dust particle. For numerical validation of the analytical results, we have taken the results obtained in equations (54) and (56). The constants $W, X, Y, Z$ is calculated in terms of Prandtl no. $\mathrm{P}_{\mathrm{r}}$, constants $\mathrm{N}_{0}, \xi_{0}, \xi_{1}$, kinematic viscosity $v$, thermal conductivity $\mathrm{k}$. In the present study we adopted the following default parametric values for some

\begin{tabular}{|c|c|c|c|c|c|c|c|c|}
\hline Fluid & $\mathbf{P}_{\mathrm{r}}$ & $\mathbf{N}_{0}$ & $\xi_{0}$ & $\xi$ & $\mathbf{W}$ & $\mathbf{X}$ & $\mathbf{Y}$ & $\mathbf{Z}$ \\
\hline Mercury & 0.015 & .1 & .01 & .02 & .00058 & $4.18 \times 10^{-7}$ & $3.69 \times 10^{-13}$ & 5.87 \\
\hline MixGas & 0.2 & .1 & .01 & .02 & $1.15 \times 10^{-6}$ & $5.75 \times 10^{-18}$ & $3.78 \times 10^{-16}$ & $9.95 \times 10^{-13}$ \\
\hline Hyd.Gas & 0.4 & .1 & .01 & .02 & $4.86 \times 10^{-7}$ & $9.4 \times 10^{-20}$ & $1.9 \times 10^{-16}$ & $2.3 \times 10^{-15}$ \\
\hline HelGas & 0.7 & .1 & .01 & .02 & $4.6 \times 10^{-6}$ & $4.8 \times 10^{-16}$ & $7.4 \times 10^{-13}$ & $9.4 \times 10^{-23}$ \\
\hline
\end{tabular}
fluid in the table has discussed step by step

Table 1. The value of the constants and parameter used in equation (54) and (55).

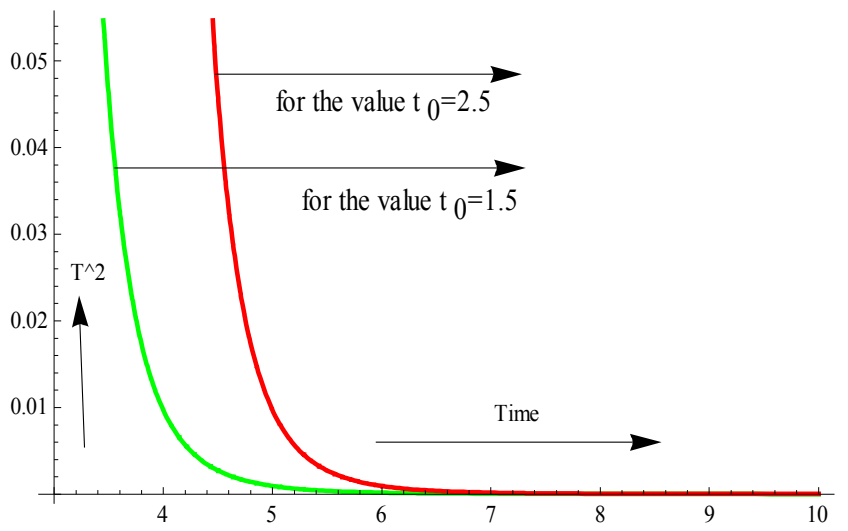

Figure 1. When $P_{r}=.01, l=0$ (for clean fluid).

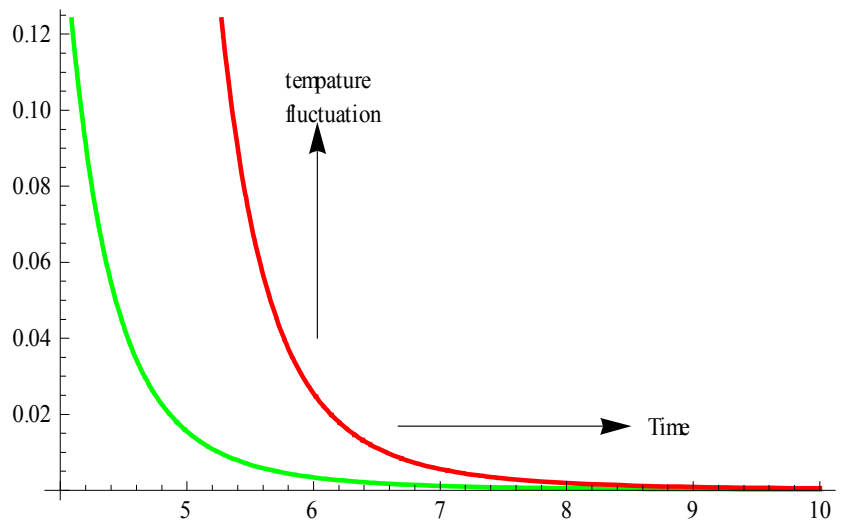

Figure 2. For $l=0.5$.

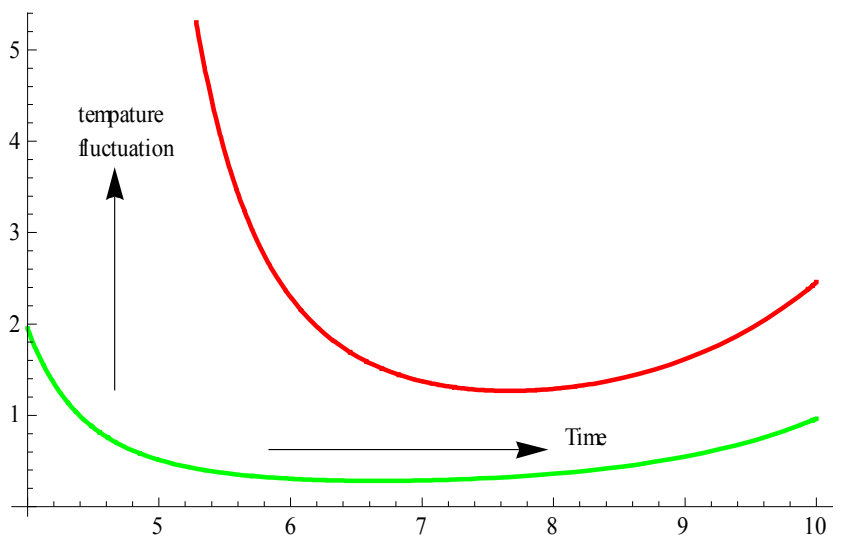

Figure 3. For $l=1.5$.

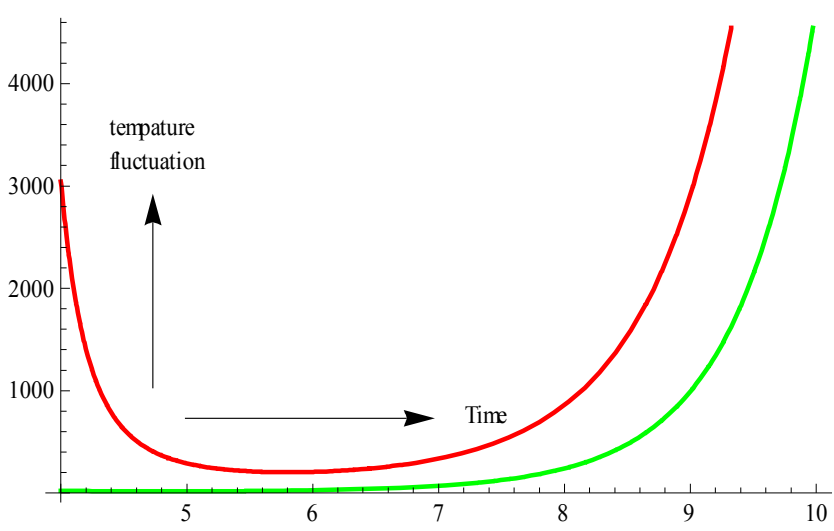

Figure 4. For $l=2.5$. 


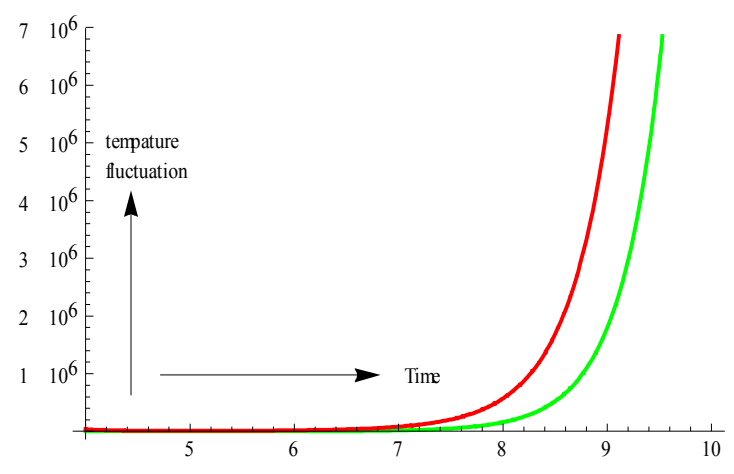

Figure 5. For $l=3.5$.

Figure 1 and Figure 2, Figure 3, Figure 4, Figure 5, represents the energy decay of temperature fluctuation for four-point correlations of equation (54). When the Prandtl no. is small as of mercury $\mathrm{P}_{\mathrm{r}}=.015$. It is observed from the above figures the energy decays more rapidly as dust particle increases from 0.5 to 3.5 that of clean fluid.

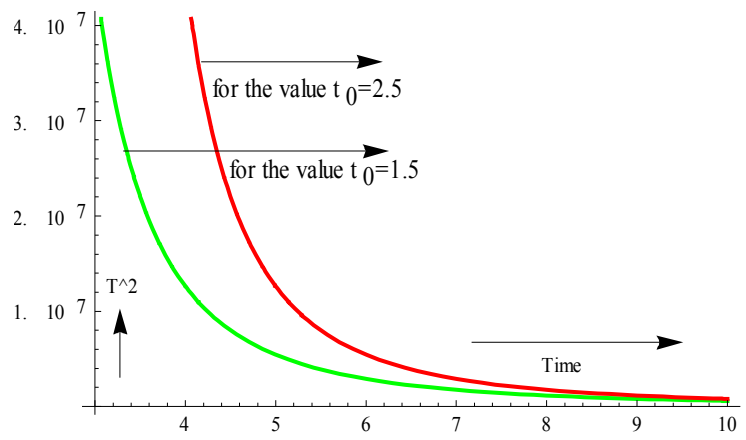

Figur 6. Energy decay for mix. of gas $p_{r}=.2$ forcleanfluid.

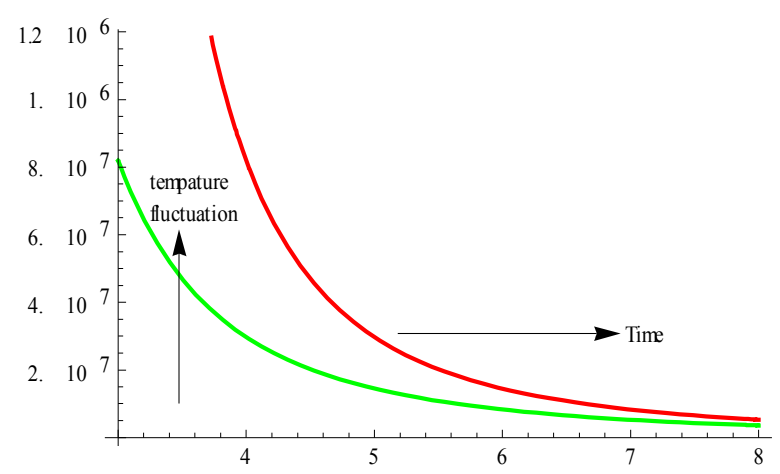

Figure 7. For $l=0.5$.

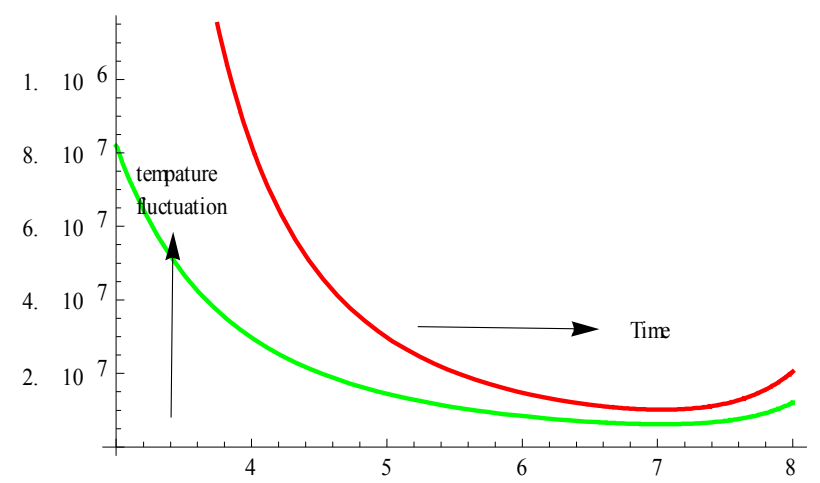

Figure 8. For $l=1.5$

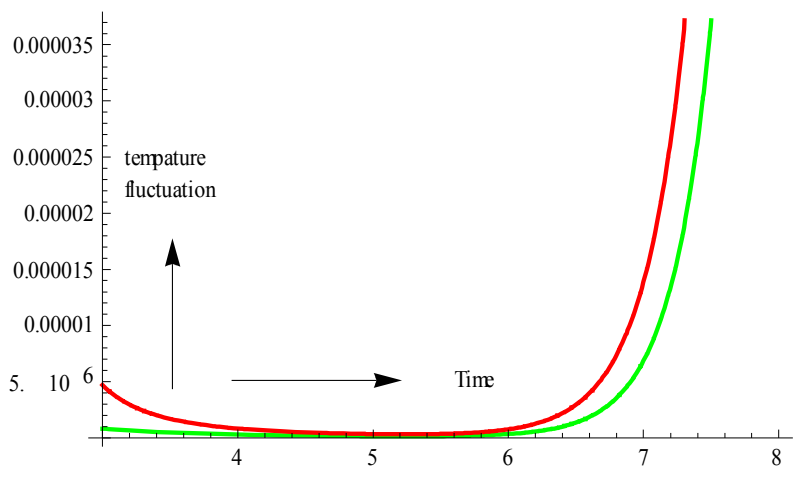

Figure 9. For $l=2.5$.

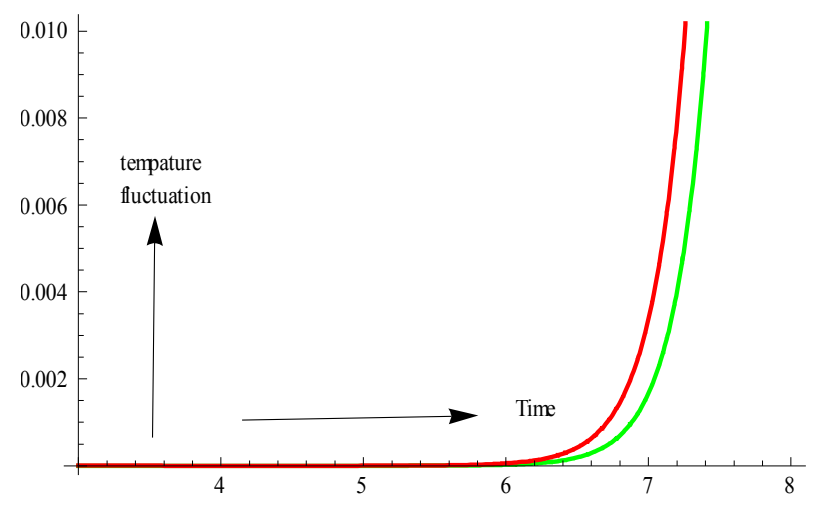

Figure 10. For $l=3.5$.

The above figures represent the energy decay of temperature fluctuation for four -point correlations of equation (54). With fixed Prandtl no. is as of mix. of gas $\mathrm{P}_{\mathrm{r}}=.2$, if we increases the quantity of 1 the energy decays rapidly more and more.

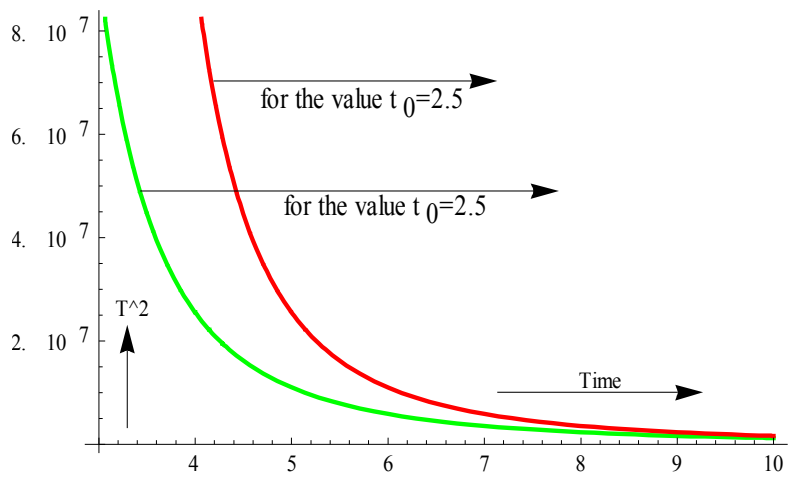

Figure 11. $P_{r}=.4, l=0$

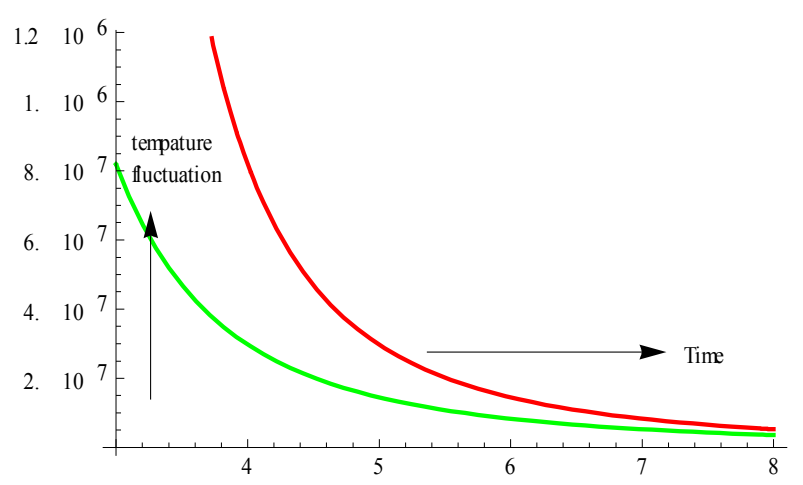

Figure 12. For $l=0.5$. 

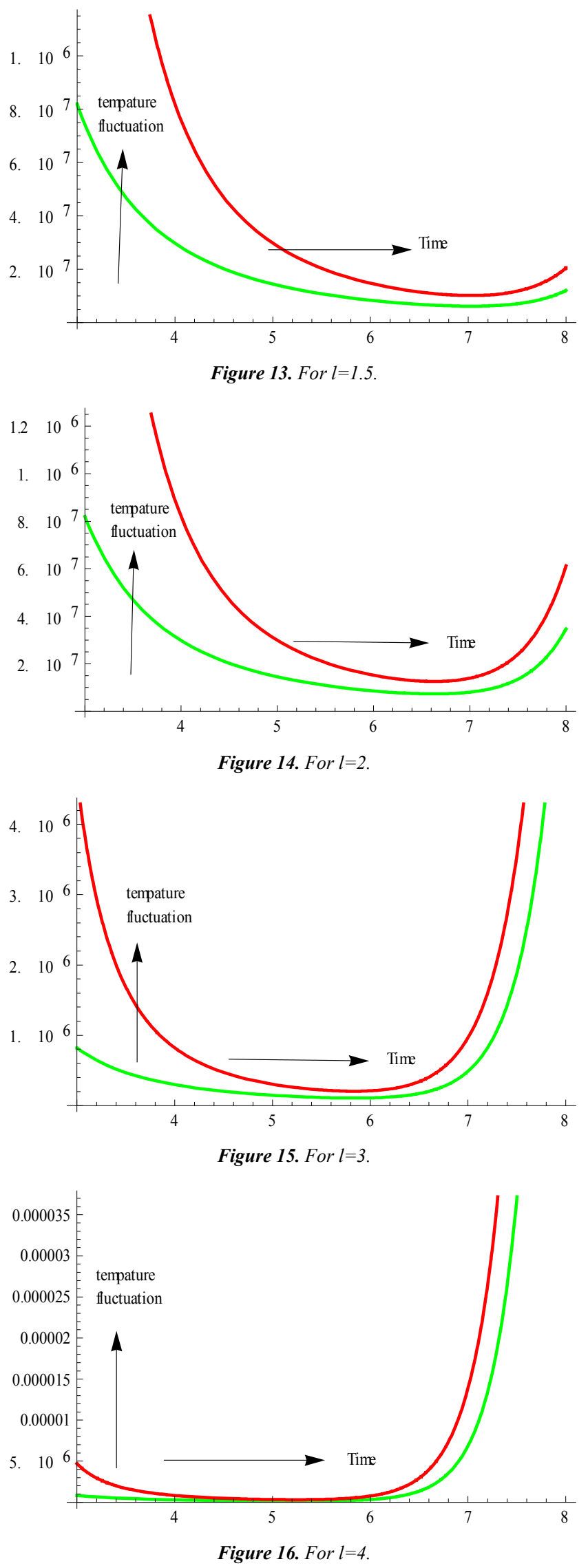

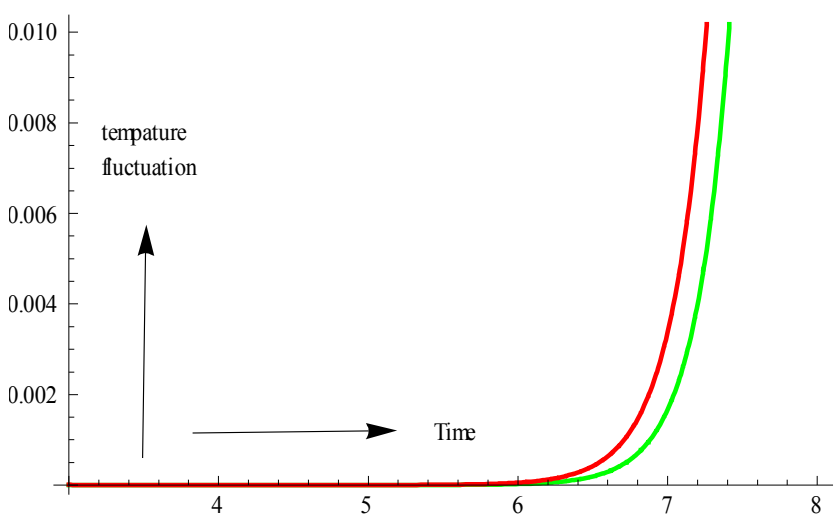

Figure 17. For $l=7$.

Figure 11 represents the energy decay of temperature fluctuation for four -point correlations of equation (54). When the Prandtl no. is as of Hyd gas $\mathrm{P}_{\mathrm{r}}=.4$ without dust particles and Figure 12- Figure 17represents the energy decay of temperature fluctuation for four -point correlations of equation (54). When the Prandtl no. is as of Hyd gas $\mathrm{P}_{\mathrm{r}}=.4$ with dust particles for $1=0.5,1.5,2,3,4$ and 7 respectively

\section{Conclusions}

Through this study we conclude thatthe temperature energy in homogeneous dusty fluid turbulent flow for fourpoint correlations decays rapidly more and more by exponential manner than that of clean fluid. It is observed that the energy decreases more rapidly as Prandtl number increases which shows in Figure 1, Figure 6 and Figure 11 in the case of clean fluid. From the resulting equations and its figures we have tried to show the effect of dust particle in the Figure 2- Figure 5; Figure 7- Figure 10; Figure 12- Figure 17 that the energy decays more faster and abruptly change than that of clean fluids the quantity of dust particle gradually increases.

\section{References}

[1] S. Corrsin, On the spectrum of isotropic temperature fluctuations in isotropic turbulence, J. Apll. Phys, Vol.22, pp 469-473, $1951 b$.

[2] ] R. G. Deissler, on the decay of homogeneous turbulence before the final period, Phys. Fluids, Vol. 1, pp 111-121, 1958.

[3] R. G. Deissler, A theory of decaying homogeneous turbulence, Phys. Fluids, Vol. 3, pp 176-187. 1960.

[4] A. L. Loeffler and R. G. Deissler, Decay of temperature fluctuations in homogeneous turbulence before the final period, Int. J. Heat Mass Transfer, Vol. 1, pp 312-324,1961.

[5] P. G. Saffman, On the stability of laminar flow of a dusty gas. J. Fluid Mech., Vol. 13 pp 120-128, 1962.

[6] N. Kishore and S. R. Singh, Statistical theory of decay process of homogeneous hydromagnetic turbulance,Astrophysics and Space Scie. Vol.104, pp 121-125, 1984. 
[7] Kishor, N. And Y. T. Golsefied, Effect of coriolis force on acceleration covariance in MHD turbulent flow of a dusty incompressible fluid. Astrophys. Space Sci., Vol.150(1), pp 89 101,1988

[8] N. Kishore and M. S. A. Sarker, Rate of change of vorticity covariance in MHD turbulent flow of dusty incompressible fluid. I. J. Energy Res. m 14, Vol. 5, pp 573-57.1990.

[9] M. A. K. Azad and M. S. AlamSarker, Decay of MHD turbulence before the final period for the case of multi-point and multi-time in presence of dust particle, Bangladesh J. Sci. Ind. Res. Vol.38(3-4), pp 151-164, 2003.

[10] . M. A. K. Azad and M. S. Alam Sarker, Decay of temperature fluctuations in homogeneous turbulence before the final period for the case of multi-point and multi-time in presence of dust particle, Rajshahi University Studies, Part-B, Journal of Science, Vol. 34, pp 37-50, 2006.

[11] M. A. K. Azad, M. S. Alam Sarker and N. I. Mondal, Decay of temperature fluctuations in dusty fluid MHD turbulence before the final period in a rotating system, Journal of Engng. and Applied Siences, Vol.1(2), pp187-194, 2006.

[12] M. A. K. Azad and M. S. Alam Sarker, Decay of temperature fluctuations in homogeneous turbulence before the final period for the case of multi-point and multi-time in a rotating system in presence of dust particle, Journal of Applied Sciences Research, Vol.4(7), ${ }_{\mathrm{pp}} 793-802,2008$.

[13] M. A. K. Azad, M. A. Aziz and M. S. Alam Sarker, First Order Reactant in Magneto-hydrodynamic Turbulence before the Final Period of Decay in presence of dust particles in a Rotating System, Journal of Physical Sciences, vol.13, pp 175190, 2009.

[14] M. S. Alam Sarker, M. A. K. Azad and M. A. Aziz, First Order Reactant in MHD Turbulence before the Final Period of Decay for the Case of Multi-Point and Multi-Time in Presence of Dust Particles. J. Phy, Sci, Vol. 13, pp21-38, 2009.

[15] . M. A. K. Azad, M. S. Alam Sarker and M. A. Aziz, Decay Law of temperature fluctuations in dusty fluid MHD Turbulence before the final Period, J. Math. Forum, Vol. XX, pp 32-48, 2007-7008-2009.

[16] M. A. K. Azad, M. H. U. Molla and M. Z. Rahman,Transport Equatoin for the Joint Distribution Function of Velocity, Temperature and Concentration in Convective Tubulent Flow in Presence of Dust Particles, Research Journal of Applied Sciences, Engineering and Technology( RJASET) Vol. 4(20), pp 4150-4159, 2012.
[17] M. A. K. Azad and Mist. Mumtahinah, Decay of Temperature fluctuations in dusty fluid homogeneous turbulence prior to final period, Research Journal of Applied Sciences, Engineering and Technology Vol.6(8), pp 1490-1496, 2013.

[18] M. A. K. Azad and Mist. Mumtahinah, Decay of Temperature fluctuations in dusty fluid homogeneous turbulence prior to the ultimate period in presence of coriolis force, Res. J. Appl. Sci. Engng. and Tech. Vol.7(10), pp 1932-1939, 2014.

[19] M. A. BkarPk, A. Malek and M. A. K. Azad, 4-point correlations of dusty fluid MHD turbulent flow in a $1^{\text {st }}$ order reaction, Global J. of Science Frontier Research: F Mathematics \& Decision Sciences Vol.15(2), version 1.0, pp 53-69, 2015.

[20] M. A. K. Azad, Abdul Malek\& M. A. BkarPk, 3-Point Distribution Functions in the Statistical Theory in MHD Turbulent flow for Velocity, MagneticTemperature and Concentration undergoing a First Order Reaction, Global J. of Science Frontier Research: A Physics and Space Science, Vol.15(2) Version 1.0,pp 13-45, 2015.

[21] M. A. K. Azad, M. Abu BkarPk, \& Abdul Malek, Effect of Chemical Reaction on Statistical Theory of Dusty Fluid MHD Turbulent Flow for Certain Variables at Three- Point Distribution Functions, Science Journal of Applied Mathematics and Statistics, Vol.3(3), pp 75-98, 2015.

[22] M. A. K. Azad, Mst. Mumtahinah \& M. Abu BkarPk, Statistical Theory of Three- Point Distribution Functions in MHD Turbulent flow for Velocity, Magnetic Temperature and Concentration in a Rotating System in presence of Dust Particles, Int. J. Scholarly Res. Gate, Vol. 3(3), pp 91-142, 2015.

[23] M. A. K. Azad,., M. H. U. Molla \& M. Z. Rahman, Transport Equatoin for the Joint Distribution Functions in Convective Tubulent Flow in Presence of Dust Particles undergoing a first order reaction, Rajshahi Univ. J. Sci. and Engng ( accepted for publication), Vol.40-42, pp31-43, 2012- 2014.

[24] M. S. Alom,M. Ali. M. A. Alim, Study the Effect of Chemical Reaction and Magnetic Field on Free Convection Boundeary Layer Flow of Heat and MassTransfer with Variable Prandtl Number. J. Sci. Res. Vol.8(1), pp41-18,2016.

[25] M. Mamun Miah, M. A. K. Azad, M. Masidur Rahman, The Effect of 1st Order Chemical Reaction in Convective Dusty Fluids Turbulent Flow for Three-Point Joint Distribution Functions, IOSR Journal of Mathematics (IOSR-JM), Vol. 12, pp 12-35, I(Jan. - Feb. 2016), 17-35, 2016. 